\title{
Numerical Simulation of Flow-Induced Vibration of Three-Dimensional Elastic Heat Exchanger Tube Bundle Based on Fluid-Structure Coupling
}

\author{
Shiqin Ai, Chao Sun $(\mathbb{D}$, Yuechan Liu $(\mathbb{D}$, and Yuelin Li \\ School of Measurement and Communication Engineering, Harbin University of Science and Technology, Harbin 150080, China \\ Correspondence should be addressed to Chao Sun; sc13579@126.com and Yuechan Liu; shirley2518120@aliyun.com
}

Received 11 August 2021; Revised 19 November 2021; Accepted 16 December 2021; Published 12 January 2022

Academic Editor: Giorgio Dalpiaz

Copyright (c) 2022 Shiqin Ai et al. This is an open access article distributed under the Creative Commons Attribution License, which permits unrestricted use, distribution, and reproduction in any medium, provided the original work is properly cited.

\begin{abstract}
The reliability of the heat exchanger tube bundle not only affects the economic efficiency of production but also relates to the normal development of production safety and health. To study the flow-induced vibration of tube bundles, a three-dimensional finite element model of heat exchange tubes and watersheds inside and outside the tubes was established to explore the flowinduced vibration characteristics of tube bundles and analyze the natural frequencies of single-span and multispan heat exchange tubes. Considering the randomness of the effective support between the tube bundle and the support plate of the heat exchanger, the natural frequency and vibration mode of the four-span tube with failure of the tube bundle support are analyzed. On this basis, the vibration caused by the two-way coupling flow between tube and tube outflow is calculated. Finally, the flow-induced vibration characteristics of the five-tube bundle with two different pitch-diameter ratios are analyzed. The calculation results show that the error between the calculated natural frequencies and the theoretical values is less than $3 \%$, and within the allowable error range, the natural frequencies of the same order decrease with the increase of the number of support failures. The vibration frequencies of single-span and multispan tube bundles are consistent with the lift and drag frequencies, the vibration displacement curves show typical Strouhal modes, and the amplitude increases with the increase of fluid velocity. Vibration displacement curves of symmetrical spans of multispan tube bundles are similar in shape and amplitude. With the increase of tube bundle spacing, the vibration characteristics become more obvious.
\end{abstract}

\section{Introduction}

With the development of energy-saving technology, the heat exchanger has been widely used in the chemical industry and has brought significant economic benefits. However, to meet the needs for production and the continuous pursuit for high thermal efficiency, the structural characteristics of heat exchanger tube bundle become more and more flexible, and the tube bundle is more prone to vibration, which leads to fatigue damage of the tube bundle, resulting in production safety accidents and posing a great threat to the personal safety of operators [1-3]. The heat exchanger not only influences vibration and ring breaking but also is the result of combined action under various working conditions [4]. It is fatal damage to the heat exchange tubes at the tube-plate hole, baffle tube hole, and U-shaped elbow, and the tube bundle breaks due to fatigue due to repeated vibration and friction. Therefore, it is necessary to study and analyze the characteristics of flow-induced vibration of heat exchanger tubes during the structural design, evaluation, and maintenance of heat exchanger equipment to ensure the reliability of the equipment.

There are many reasons for vibration, but the most important ones are fluid-induced vibration and dynamic mechanical vibration propagated by heat exchanger connectors [5]. Flow-induced vibration failure of fixed tubeplate heat exchangers has always been a hot issue in heat exchanger research. This kind of damage will cause the damage of parts in the heat exchanger structure to varying degrees, especially in the heat exchange tubes, baffles, and important joints $[6,7]$. Previous studies show that vortex shedding plays a leading role in tube bundle vibration [8]. 
When the shell-side fluid flows through the tube bundle, a vortex of alternating shedding will be formed at the back of the tube bundle, which will generate periodic alternating stress on the cylinder and break the original safe state of the tube bundle [9-12]. Under the influence of fluid scour for a long time, the energy keeps accumulating and exceeds the safe critical value, resulting in an explosion. The phenomenon of flow-induced vibration in axial flow around flow structure was discovered in late 1950, but it has not received enough attention [13]. In 1986, when the fifth installation company of the Ministry of Nuclear Industry was testing the airtightness of the heat exchanger in the first chemical plant of a petrochemical general plant, an explosion occurred suddenly when the air pressure reached $3.5 \mathrm{MPa}$, resulting in the death of four on-site workers. In 2012, Unit 3 of San Onofre Nuclear Power Plant in the United States caused excessive vibration of $U$-shaped heat transfer tube of steam generator, which caused friction and wear of the heat transfer tube and nuclear leakage, and the reactor was shut down, causing a serious production safety incident [14].

Lai pointed out in his article that only when the velocity is much higher than the normal velocity, the longitudinal flow-induced vibration should be considered [15]. Under the normal transverse flow velocity, greater vibration may be caused, and the damage to the structure is the greatest. People pay more attention to the damage caused by transverse flow-induced vibration. Jin et al. pointed out the shortcomings in the research of a single flexible tube through the water tunnel experiment [16]. Zhu studied the two-way fluid-structure coupling numerical simulation of internal and external liquid-filled tube beam induced vibration and found that the numerical simulation results of one-way fluid-structure coupling were not very reliable [17]. Zhao et al. analyzed the interaction between two adjacent tube bundles and obtained the relationship between the pitch-diameter ratio and the displacement of the two adjacent tube bundles [18]. Liu et al. studied the vibration characteristics of linear and staggered tube bundles through numerical calculation and experimental comparative analysis [19]. Feng et al. analyzed the fluid force and vibration response characteristics of different elastic tube bundle models by using the three-dimensional numerical model of the fluid-structure coupling system of square aligned elastic tube beam [20]. Zhou simulated and analyzed the problem of incompressible fluid flowing around a cylinder under the condition of two-dimensional elastic support and pointed out that the maximum amplitude of the cylinder occurs when the ratio of the natural frequency of the cylinder to Karman vortex shedding frequency is close to $1: 1$ [21]. In the past, studies on the flow-induced vibration of heat exchanger tube bundles, theoretical analysis, experimental analysis, and numerical simulation were carried out separately or combined, and more accurate and reliable results are obtained, but there are few studies on the flow-induced vibration of five-tube bundles with multiple tube bundles and different nodal diameter ratios. According to the analysis of fluid elastic instability, Liu and others put forward a new instability model based on other people's research and pointed out that the improved model has the advantages of better calculation accuracy and less dependence on experimental data [22], but it does not analyze the flow-induced vibration of elastic tube bundle caused by vortex shedding, which has certain limitations.

Local melting occurred in American Experimental Breeder Reactor No.1, which was mainly caused by fretting wear caused by flow-induced vibration. The problem of flow-induced vibration in nuclear reactors was paid attention to and then gradually extended to various fields of heat exchange equipment production industry, and the production safety work was well developed. The existing research mainly focuses on the analysis of three-dimensional flow-induced vibration of a single tube bundle and the discussion of flow-induced vibration of two tube bundles. At present, most simulation calculations consider the rigid tube or calculate the one-way fluid-structure coupling, but the analysis of two-way fluid-structure coupling in the case of multiple tube bundles has not been published in the literature. In this paper, the flow-induced vibration of the heat exchange tube bundle is analyzed by COMSOL. At the same time, the natural frequencies of tube bundles under different failure supports are studied. The two-way fluid-structure coupling method is used not only to analyze the flow-induced vibration of a single tube bundle with elastic support but also to calculate the vortex-induced vibration of fivetube bundles with two different spacing. As a consequence, it provides a reference for studying the flow-induced vibration of multiple tube bundles by using the two-way fluidstructure coupling method.

\section{Natural Frequency of Heat Exchange Tube}

2.1. Calculation of Theoretical Value. Solving the vibration problem of heat exchange tubes can be equivalent to the vibration problem of continuous beams. According to the natural frequency calculation of the heat exchange tube bundle, the theoretical calculation formulas are as follows [23]:

$$
f_{n}=35.3 \lambda_{n} \sqrt{\frac{E\left(D^{4}-d^{4}\right)}{m l^{4}}} .
$$

$\lambda_{n}$ is determined by the following transcendental equation, where $\mathrm{N}$ is the number of spans:

$$
\cos \frac{n \pi}{N}=\frac{(1 / \tan h \lambda)-(1 / \tan \lambda)}{(1 / \sin h \lambda)-(1 / \sin \lambda)} .
$$

When the number of frequency bands is odd,

$$
n=0,1,2, \ldots, N-1 \text {. }
$$

When the number of frequency bands is even,

$$
n=1,2,3, \ldots, N \text {. }
$$

The mass per unit length $m$ includes the mass of empty tubes per unit length $m_{t}$, the mass of water in heat exchange tubes per unit length $m_{i}$, and the mass of water outside heat exchange tubes per unit length $m_{o}$. Look up the table to 
know that the tube sheets at both ends are fixed and supported, and the frequency constant $\lambda_{n}$ of straight tubes with equal span is 22.396. The material of the heat exchange tube is $0 \mathrm{Cr} 19 \mathrm{Ni} 9$ steel, and the specific parameters are shown in Table 1.

The failure of tube bundle at different positions can be calculated according to the calculation method in reference 26 , and the basic principle is as follows.

The natural frequency is calculated by the allowable function of displacement boundary conditions satisfied under different failure conditions. The failure model dimensions are shown in Figure 1.

If the leftmost or rightmost of the middle three simply supported supports fails, then the admissible functions straight tube is as follows:

$$
\begin{aligned}
& \varphi_{1}(x)=x^{2} \cdot(x-L)^{2} \cdot(x-l) \cdot(x-2 l), \\
& \varphi_{2}(x)= \begin{cases}1-\cos \left(\frac{2 \pi x}{l}\right), & 0 \leq x \leq 2 l, \\
1-\cos \left[\pi\left(\frac{x-2 l}{l}\right)\right], & 2 l \leq x \leq L .\end{cases}
\end{aligned}
$$

Only the support in the middle fails; currently, the admissible function is

$$
\begin{aligned}
& \varphi_{1}(x)= x^{2} \cdot \leq(x-L)^{2} \cdot(x-l) \cdot(x-3 l), \\
& \varphi_{2}(x)= \begin{cases}1-\cos \left(\frac{2 \pi x}{l}\right), & 0 \leq x \leq l, \\
1-\cos \left[\pi\left(\frac{x-l}{l}\right)\right], & l \leq x \leq 3 l, \\
1-\cos \left[2 \pi\left(\frac{x-3 l}{l}\right)\right], & 3 l \leq x \leq L .\end{cases}
\end{aligned}
$$

The admissible function when only the leftmost or rightmost support is valid:

$$
\begin{aligned}
& \varphi_{1}(x)=x^{2} \cdot(x-L)^{2} \cdot(x-l), \\
& \varphi_{2}(x)= \begin{cases}1-\cos \left(\frac{2 \pi x}{l}\right), & 0 \leq x \leq l, \\
1-\cos \left[2 \pi\left(\frac{x-l}{3 l}\right)\right], & l \leq x \leq L .\end{cases}
\end{aligned}
$$

According to (7) and (8), combining the admissible functions in different situations, above all, the generalized stiffness matrix and generalized mass matrix of the system are obtained. The value of $\lambda$ can be obtained by formula (12), and the natural frequency in the case of failure can be solved by bringing it into formula (1).

$$
\begin{aligned}
k_{i j} & =k_{j i}=\int_{0}^{L} E I(x) \varphi_{i}^{\prime \prime}(x) \varphi_{i}^{\prime \prime}(x) \mathrm{d} x, \\
m_{i j} & =m_{j i}=\int_{0}^{L} m(x) \varphi_{i}(x) \varphi_{j}(x) \mathrm{d} x,
\end{aligned}
$$

$$
|\mathbf{K}-\lambda \mathbf{M}|=0 .
$$

Among them, $\varphi_{i}(x)$ is the hypothetical admissible function of each order, and $i, j$ represent the order.

2.2. Single-Span Tube Bundle. The calculation model is shown in Figure 2. Considering the influence of the supporting conditions at the heat exchange tube, its structure is reasonably simplified. That is, the heat exchange tube is regarded as a straight tube. In addition, the tube sheets at both ends are regarded as fixed supports, and the fluid medium inside the tube is water.

Zhang et al. carried out fluid-structure coupling modal analysis on $U$-shaped liquid-filled pipeline. The results show that, with the increase in modal order, the natural frequency tube bundle gradually increases, while the natural frequency tube bundle without coupling calculation is greater than that of fluid-structure coupling state [24]. In this paper, the natural frequency of a single-span tube is analyzed by the numerical simulation method. Furthermore, the first five natural frequencies are calculated. To ensure the accuracy of calculation and improve the accuracy of calculation, the fluid domain and single-span tube bundle structure are divided into hexahedral meshes, which can easily fit the boundary of the region. The maximum element is $24 \mathrm{~mm}$, the minimum element is $0.24 \mathrm{~mm}$, the maximum element growth rate is 1.3 , and the total number of meshes is 44980 . The meshing results are shown in Figure 3. The calculation results are listed in Table 2.

The calculation results show that the errors between the theoretical value of the natural frequency of single-span tube and the natural frequency calculated by numerical simulation gradually increase with the increase of order, and the maximum error of the first five order frequencies is about $1.34 \%$, which is within the allowable range. The calculation results are effective, and the calculation of the natural frequency for single-span tube by numerical simulation will not cause great errors.

2.3. Equal Span Heat Exchange Tube Bundle. In the actual production design, the thermal expansion of the tube and the convenience of installation are considered. There is a gap between the heat exchange tube and the support, and the tube bundle will have support without contact after the fluid flows around, which is called ineffective support. Assuming that all supports are effective supports, the four-span heat exchange tubes are calculated according to the natural frequency calculation theory in 2.1 , and $\lambda_{n}$ is 11.514 . The material of the heat exchange tube is unchanged, the baffle of the four-span tube is simplified as a simple support, and the tube sheets at both ends are simplified as fixed support (see Figure 1). The total length of the four-span tube is $L=1.2 \mathrm{~m}$, the length of each span is $1=0.3 \mathrm{~m}$, the inner diameter is $d=15 \mathrm{~mm}$, and the outer diameter is $D=16 \mathrm{~mm}$. The theoretical calculation results and numerical simulation results of natural frequencies are shown in Table 3.

It can be seen from Table 3 that the error of natural frequency obtained by the two calculation methods is still 
TABle 1: Physical parameters of the tube bundle.

\begin{tabular}{lcccccc}
\hline Material & Density & Elastic modulus $(\mathrm{GPa})$ & Poisson's ratio & Tube length & Inner diameter $(\mathrm{mm})$ & Outer diameter $(\mathrm{mm})$ \\
\hline 0Cr19Ni9 & $7900 \mathrm{~kg} / \mathrm{m}^{3}$ & 195 & 0.247 & $1.2 \mathrm{~m}$ & 15 & 16 \\
\hline
\end{tabular}

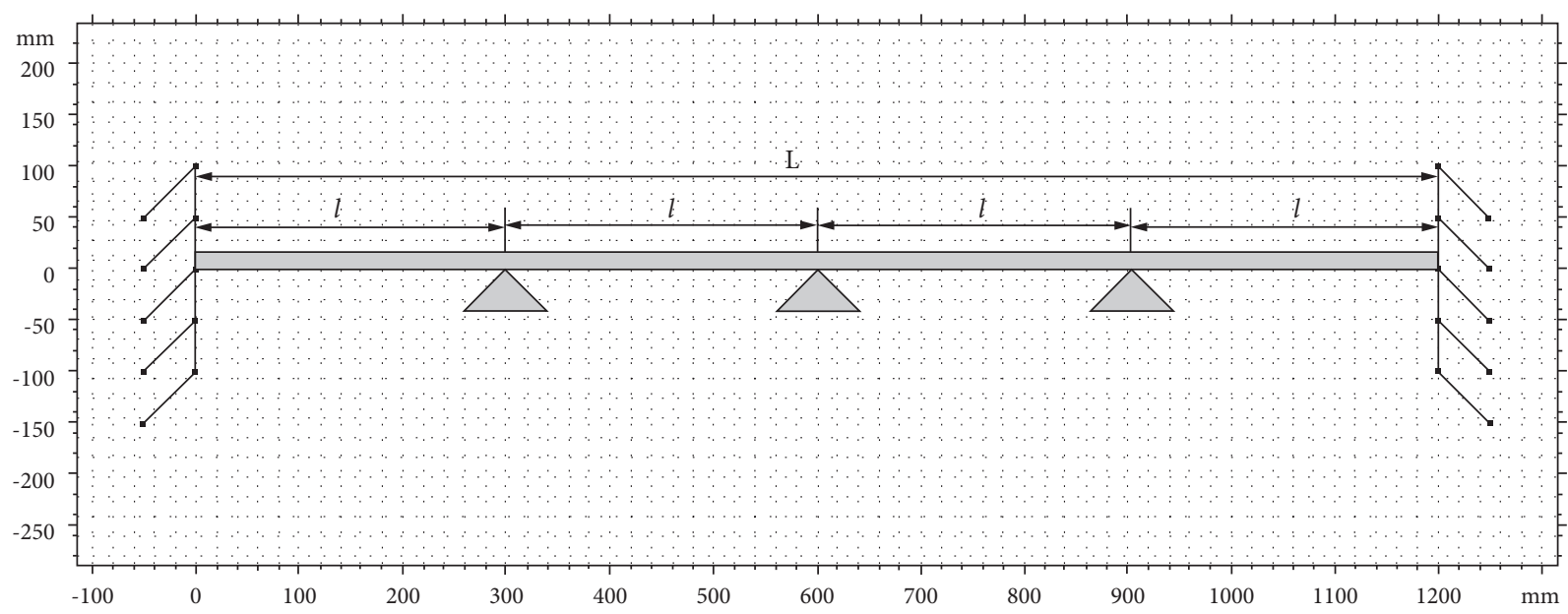

Figure 1: Four-span tube bundle model with the natural frequency.

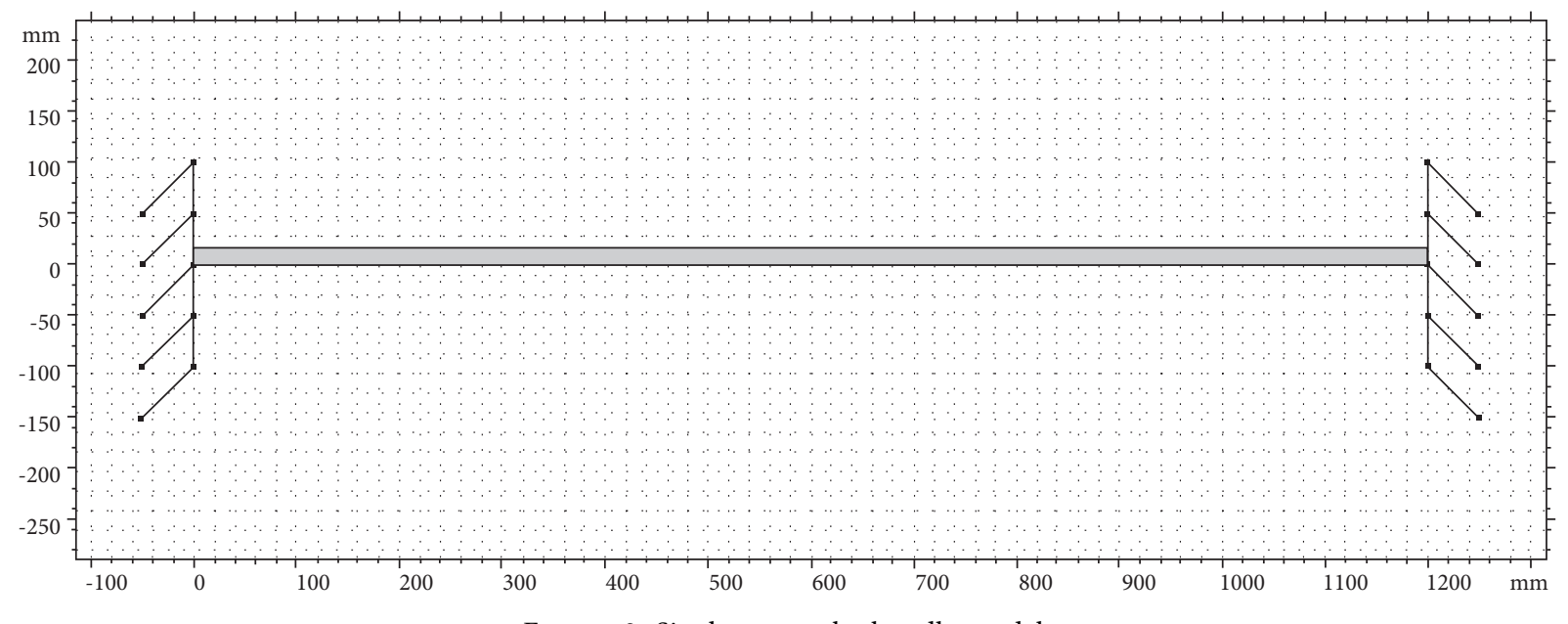

FIgURE 2: Single-span tube bundle model.

less than 3\%. The error between theoretical calculation value and numerical calculation value of the first-order natural frequency of the four-span tube bundle is about 3\%, which is different from that of the single-span tube, but the error is within the allowable range. So, this method can be used to calculate the natural frequency of multispan tubes. The natural frequency of multispan tube is generally higher than that of single-span tube, which shows that the number of tube bundle spans will have an impact on frequency calculation.

2.4. Unequal Span Heat Exchange Tube Bundle. When the heat exchanger equipment runs in a common and regular way, all parts of the equipment reach the best state, which has high reliability and lasting nature. With the increase of running time, the equipment will be damaged to varying degrees, resulting in reliability and stability that cannot be guaranteed. When the baffle of a supporting heat exchange tube in the multispan tube bundle fails, the phenomenon of nonequal span support is effective, and there is a big hidden danger in production safety. Tan, Li, and others used the numerical simulation method to study the influence of three-dimensional tube bundle support failure on the natural frequency of heat exchange tubes and decided that the failure of heat exchange tube support will significantly reduce the natural frequency of heat exchange tubes [25]. Taking the four-span tube bundle as an example, the failure support baffle is replaced by number 0 , and the effective support baffle is replaced by number 1 [26]. The natural frequency of nonequal span straight tube bundle is calculated and carefully studied. Additionally, there are three different failures at different positions, namely, the " 011 " type; “101” type; “100” type (see Figure 4). 

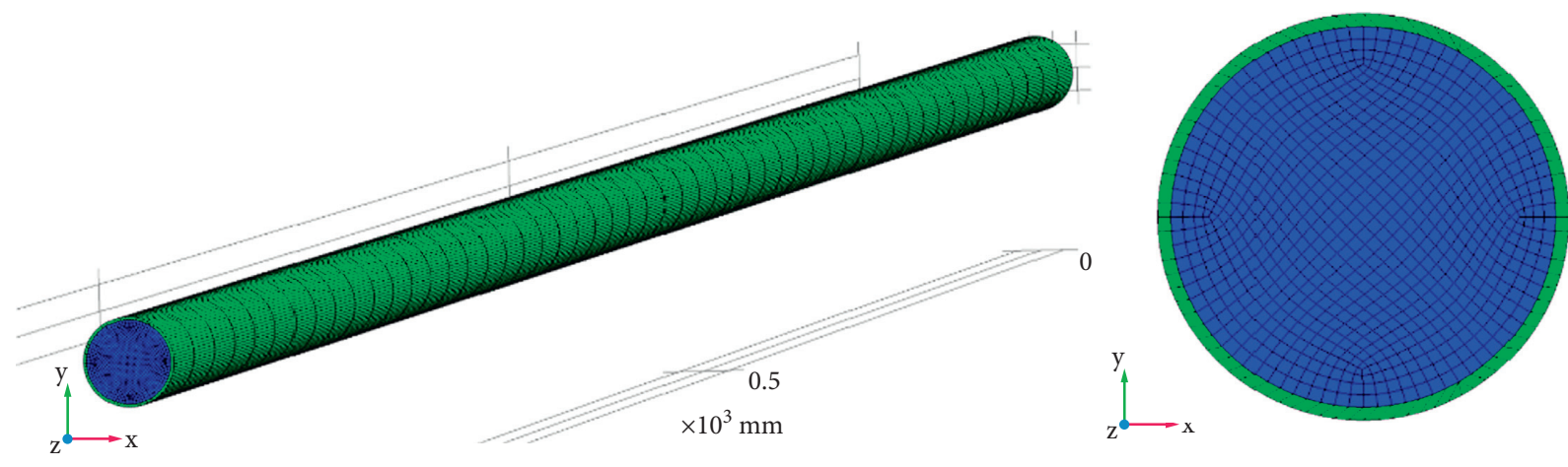

Figure 3: Schematic diagram of tube bundle structure and end face grid with internal flow.

TABLE 2: Theoretical and numerical values for the natural frequency of single-span tube.

\begin{tabular}{lccc}
\hline Order & Theoretical value $(\mathrm{hz})$ & Numerical calculation value $(\mathrm{hz})$ & Error $(\%)$ \\
\hline First-order frequency & 50.189 & 50.596 & 0.81 \\
Second-order frequency & 139.38 & 139.12 & 0.19 \\
Third-order frequency & 273.25 & 271.88 & 0.50 \\
Fourth-order frequency & 451.70 & 447.68 & 0.89 \\
Fifth-order frequency & 674.76 & 665.70 & 1.34 \\
\hline
\end{tabular}

TABLE 3: Theoretical value and numerical calculation value for natural frequency of four-span tube.

\begin{tabular}{lccc}
\hline Order & Theoretical value $(\mathrm{hz})$ & Numerical calculation value $(\mathrm{hz})$ & Error $(\%)$ \\
\hline First-order frequency & 400.4 & 412.90 & 3.12 \\
Second-order frequency & 536.2 & 550.78 & 2.72 \\
Third-order frequency & 692.8 & 708.20 & 2.22 \\
Fourth-order frequency & 778.1 & 792.77 & 1.89 \\
Fifth-order frequency & 1489.9 & 1504.4 & 0.97 \\
\hline
\end{tabular}

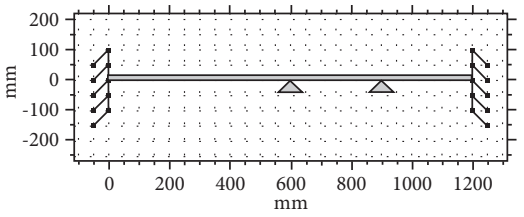

(a)

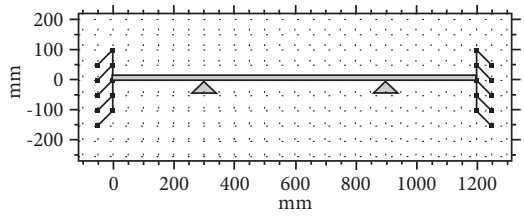

(b)

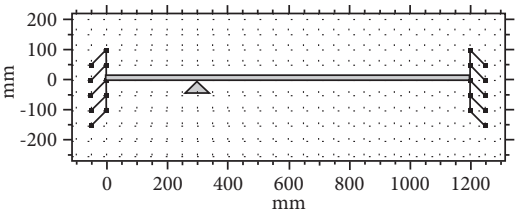

(c)

Figure 4: Unequal span tube bundle model diagram. (a) “011” type. (b) “101" type. (c) "100" type.

Numerical simulation is used to calculate the natural frequencies of the first four orders of multispan tube bundle with three failure modes, and the vibration mode diagrams under the corresponding failure states are obtained. The calculation results of natural frequencies are shown in Table 4 .

According to the calculation results in Table 4, the natural frequency of multispan tube bundles with different failure positions is different under the same number of failures supports, and the failure natural frequency of the second support point at the position is less than that of the first support point. The natural frequency of multispan tube bundle decreases with the increase of support failure number and increases with the increase of support orders. Vibration modes of different failure models are shown in Figures 5-7.

In failure modes at different positions, studies revealed that the maximum displacement of the first mode occurs at the failure position. With the increase of calculation order, the deformation of tube bundle gradually diffuses from the failure support to the whole tube bundle, and the deformation at different positions is different.

\section{Flow-Induced Vibration of Heat Exchange Tubes}

3.1. Two-Way Coupling under the Action of Single-Span Tube Bundle Water. The tube bundle of the heat exchanger will change the shape under the action for external velocity, and the vortex shedding at the tail of the tube bundle will put into action force on the tube bundle. When the transverse amplitude of a single tube bundle reaches the highest possible value, the locking in the lift direction occurs. In addition, the vortex shedding frequency is equal to the natural frequency of the tube bundle structure, of which the vibration begins to 
TABLE 4: Calculation results of natural frequencies different failure modes.

\begin{tabular}{lccccc}
\hline "011" type & & \multicolumn{2}{c}{ "101” type } & \multicolumn{2}{c}{ "100" type } \\
Frequency $(\mathrm{hz})$ & Mode characteristic & Frequency $(\mathrm{Hz})$ & Mode characteristic & Frequency (Hz) & Mode characteristic \\
\hline 169.86 & First order & 148.58 & First order & 79.960 & First order \\
422.48 & Second order & 412.90 & Second order & 223.04 & Second order \\
550.78 & Third order & 628.41 & Third order & 434.06 & Third order \\
739.61 & Fourth order & 708.20 & Fourth order & 638.94 & Fourth order \\
\hline
\end{tabular}

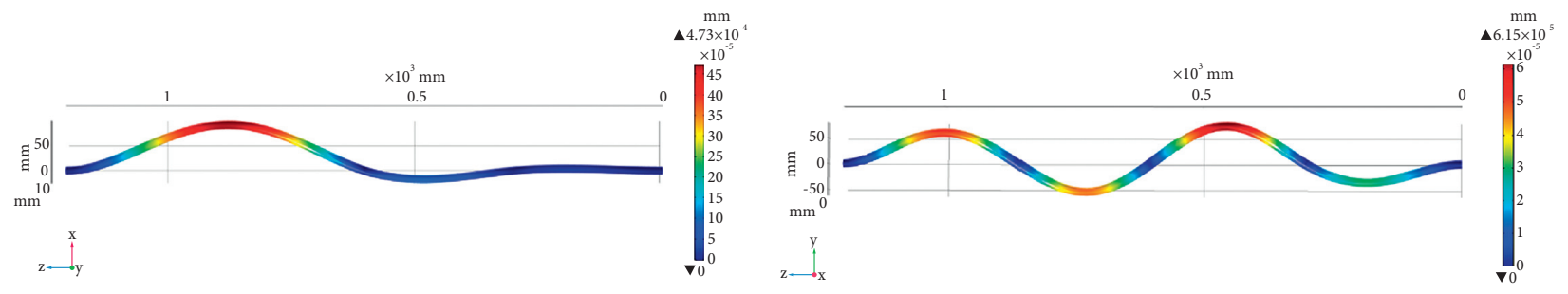

(a)

(b)

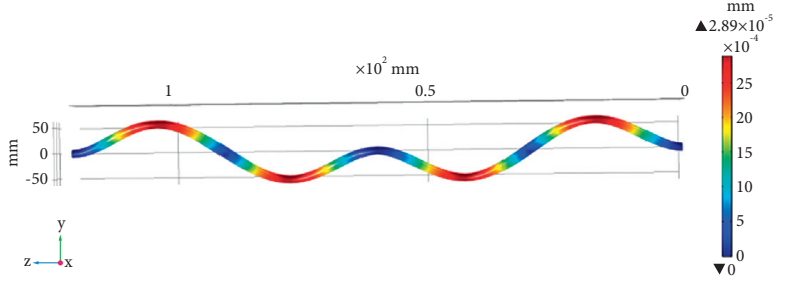

(c)

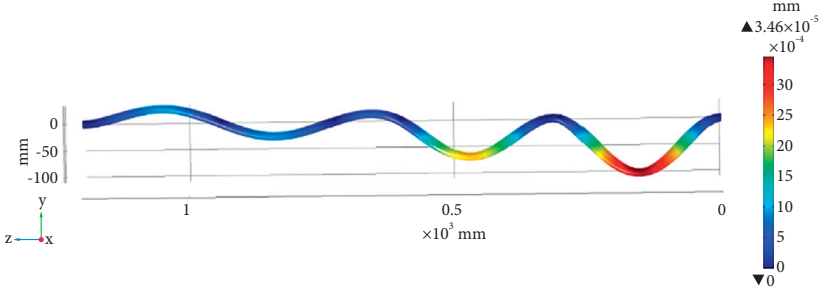

(d)

Figure 5: "011" mode. (a) First order. (b) Second order. (c) Third order. (d) Fourth order.

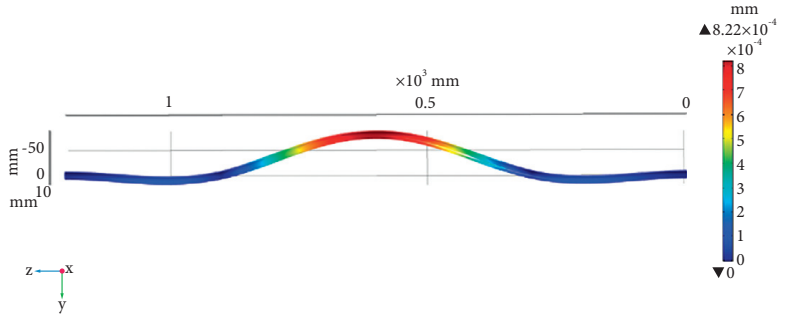

(a)

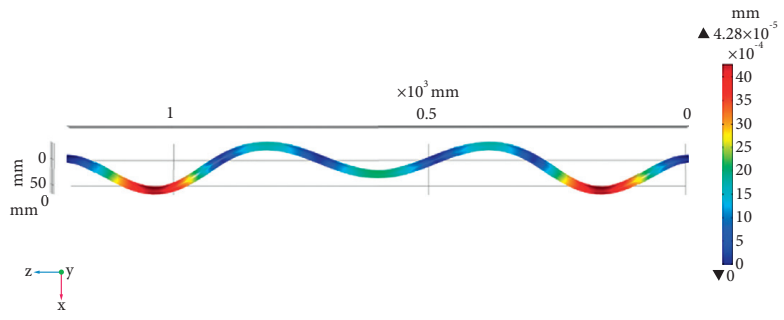

(c)

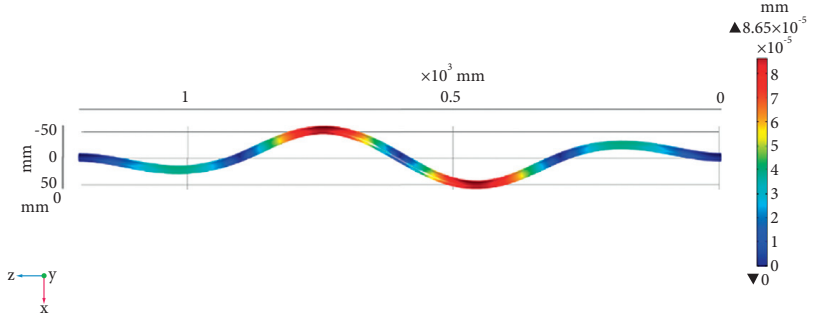

(b)

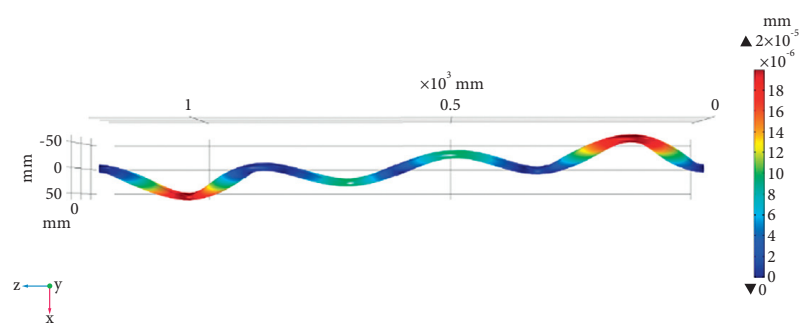

(d)

Figure 6: "101" mode. (a) First order. (b) Second order. (c) Third order. (d) Fourth order.

affect the flow field structure, resulting in a resonance phenomenon. There is basically no recirculation zone in the area far away from the tail of the tube bundle, while the motion trajectory is an " 8 " shape with a frequency ratio of 2 in the direction of drag and lift. For the trajectory of multiple tube bundles with different spacing, when the spacing tube bundles are dense, the tube bundles will influence each other, and the trajectory will be disordered. When the distance between the tube bundles is large, the influence between the tube bundles is small, which leads to the large vibration of the tube bundles in the flow direction and transverse direction, and the motion trajectory presents an " 8 " shape. At the same time, the resistance makes the motion trajectory bend to the downstream direction. Fluid-structure 


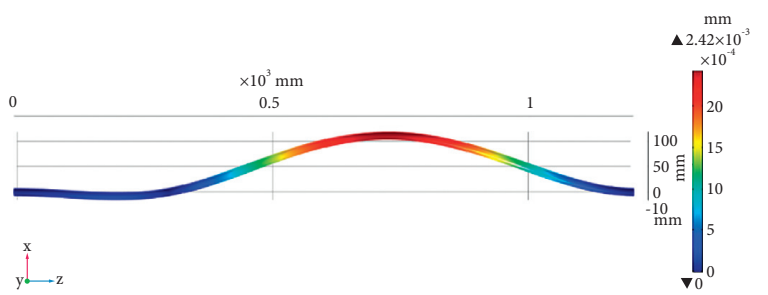

(a)

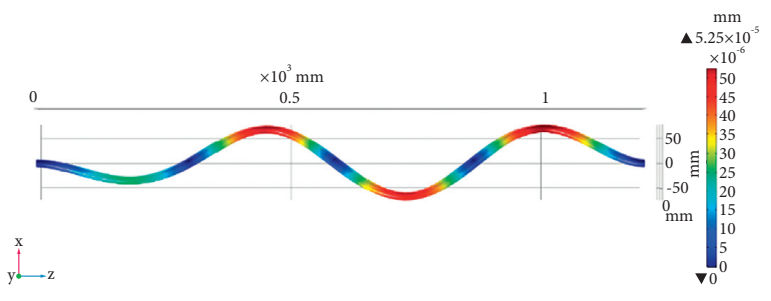

(c)

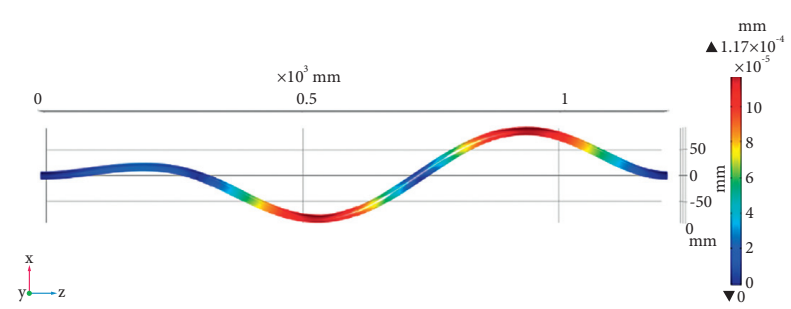

(b)

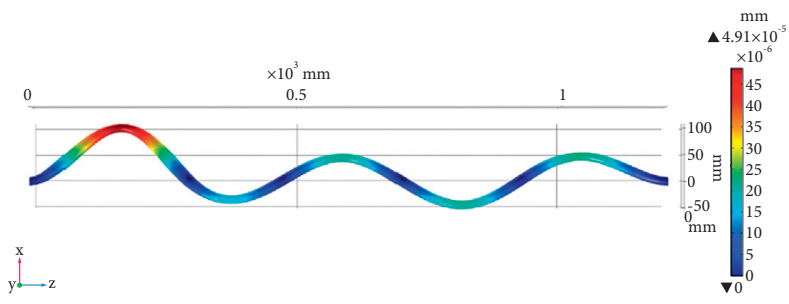

(d)

Figure 7: "100" mode. (a) First order. (b) Second order. (c) Third order. (d) Fourth order.

coupling is closely related to many engineering problems from various aspects and can solve more complex engineering problems, so it is of great significance to study heat exchangers by using the fluid-structure coupling method [27]. Through Khushnood and Nizam's experimental study and analysis, it is pointed out that the vibration amplitude of the tube bundle is larger when there is flow in the tube, and turbulence is the main excitation mechanism causing vibration in the tube within a certain range of shell-side velocity [28].

To study the flow-induced vibration of a three-dimensional tube bundle, the flow-induced vibration of a singlespan tube bundle was analyzed by using the bidirectional coupling data transfer method at the velocity of $4 \mathrm{~m} / \mathrm{s}$ in the outer basin velocity. In practical engineering activities, the heat transfer efficiency of the heat exchange tube bundle under the action of turbulence has higher advantages and plays an important role. When the transverse velocity is $4 \mathrm{~m} / \mathrm{s}$. According to Reynolds number $\operatorname{Re}=\rho U D / \mu, \operatorname{Re}=6.4 \times 10^{4}$ is in the subcritical region of vortex shedding, turbulent vortex with periodic alternate shedding will be produced at the tail of the heat transfer tube bundle, and the vibration of the tube bundle is affected by the turbulent vortex. Therefore, it is meaningful to analyze the tube bundle at the transverse velocity of $4 \mathrm{~m} / \mathrm{s}$ in order to understand the flow-induced vibration of the tube bundle in the subcritical region. Both ends of the tube bundle are fixed and supported, and the material of the heat exchange tube remains unchanged. Heat exchanger tube length is $L=0.5 \mathrm{~m}$, inner diameter is $d=15 \mathrm{~mm}$, and outer diameter is $D=16 \mathrm{~mm}$. To make sure of the full development of the flow field, the distance between the inlet of the flow field and the center of the cylinder is $2 \mathrm{D}$, the width of the flow field is 10D, and the distance between the outlet of the flow field and the center of the cylinder is $8 \mathrm{D}$.

The flow field and single-span tube bundle structure are divided by the hexahedral structured grid. Because of the large smooth change of something between two points for velocity and pressure near the wall, this paper refines the grid near the wall to improve the calculation quality of being very close to the truth or true number. We use four kinds of meshes with the number of meshes $42728,69685,81104$, and 115238 , respectively, to test the independence of meshes, so as to eliminate the influence of the number of meshes on the results and make the results more accurate. Through comparative analysis, it is known that the lifting frequency and the Strouhal number of the 81104 mesh elements vary from those of the 115238 mesh elements by less than $0.5 \%$, as shown in Table 5 .

Therefore, to keep a well-balanced compromise among computational time and solution accuracy, the third grid element used in the calculation domain is about 81104, and the grid division result is shown in Figure 8. Figure 9 shows the lift frequencies for different grid numbers.

The model is used to calculate the corresponding Strouhal numbers at different Reynolds numbers of $2 \mathrm{~m} / \mathrm{s}$, $4 \mathrm{~m} / \mathrm{s}, 6 \mathrm{~m} / \mathrm{s}$, and $17.5 \mathrm{~m} / \mathrm{s}$ and compare them with other people's results [29]. As shown in Figure 10, the calculated results are consistent with other people's experimental results.

It may be noticed from Table 6 that the error between Strouhal numbers corresponding to different Reynolds numbers and other results is less than $3.18 \%$, which has a good correspondence with the reference data.

According to the Reynolds number formula $\operatorname{Re}=\rho v d / \mu$, the Reynolds number $6.4 \times 10^{4}$ at the velocity of $4 \mathrm{~m} / \mathrm{s}$ is obtained, of which watershed is characterized by turbulence. The $\mathrm{k}-\varepsilon$ turbulence model can meet the calculation requirements, and the calculation time step is $5 \times 10^{-4} \mathrm{~s}$. The transient numerical simulation of two-way fluid-structure interaction under the action of single-span tube flow is carried out. Figure 11 shows the displacement and deformation nephogram of the tube bundle.

As can be seen from Figure 11, the heat exchange tubes swing back and forth relative to the ZX plane, and the displacement in the middle of the tube bundle is the largest. The motion trajectory is consistent with Figure 12, showing an " 8 " shape. 
TABLE 5: Results of grid independency.

\begin{tabular}{lccccc}
\hline Grid & Quantity & $\overline{\mathrm{C}_{d}}$ & $\overline{C_{l}}$ & Lift frequency $(\mathrm{hz})$ & \\
\hline 1 & 42728 & 2.19 & 0.108 & 60 & 5.82 \\
2 & 69685 & 1.863 & 0.119 & 51.82 & 0.207 \\
3 & 81104 & 1.721 & -0.02 & 49.75 & 0.199 \\
4 & 115238 & 1.728 & -0.027 & 50 & 0.200 \\
\hline
\end{tabular}

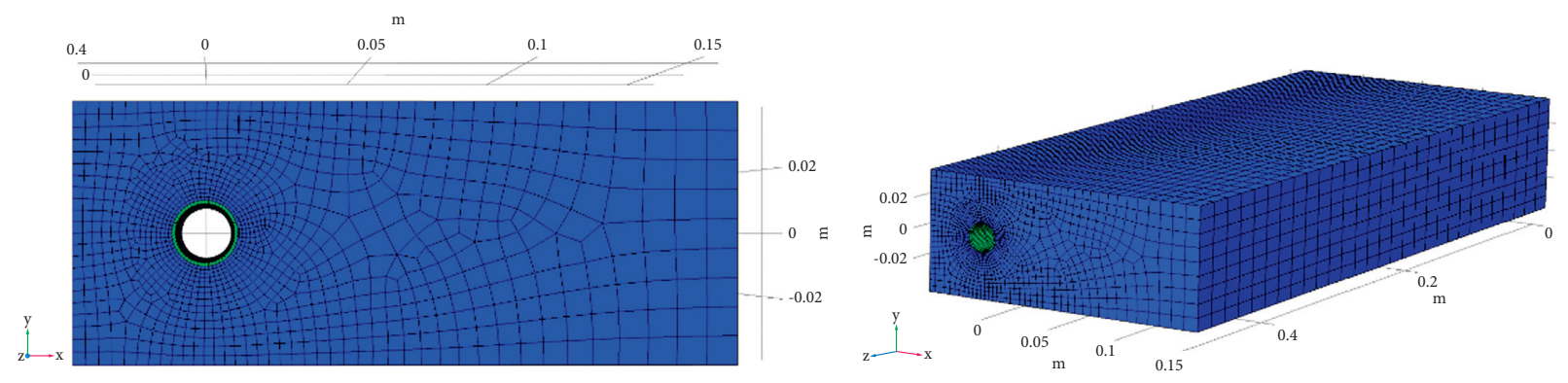

FIGURE 8: Schematic diagram of grid division.

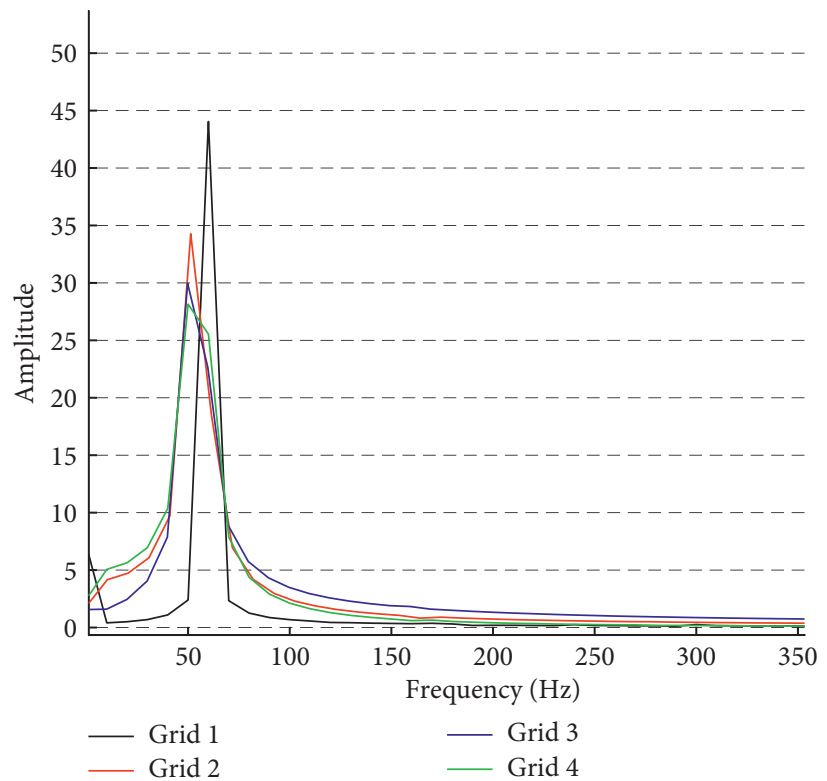

FIgURE 9: Lift frequency of different grids.

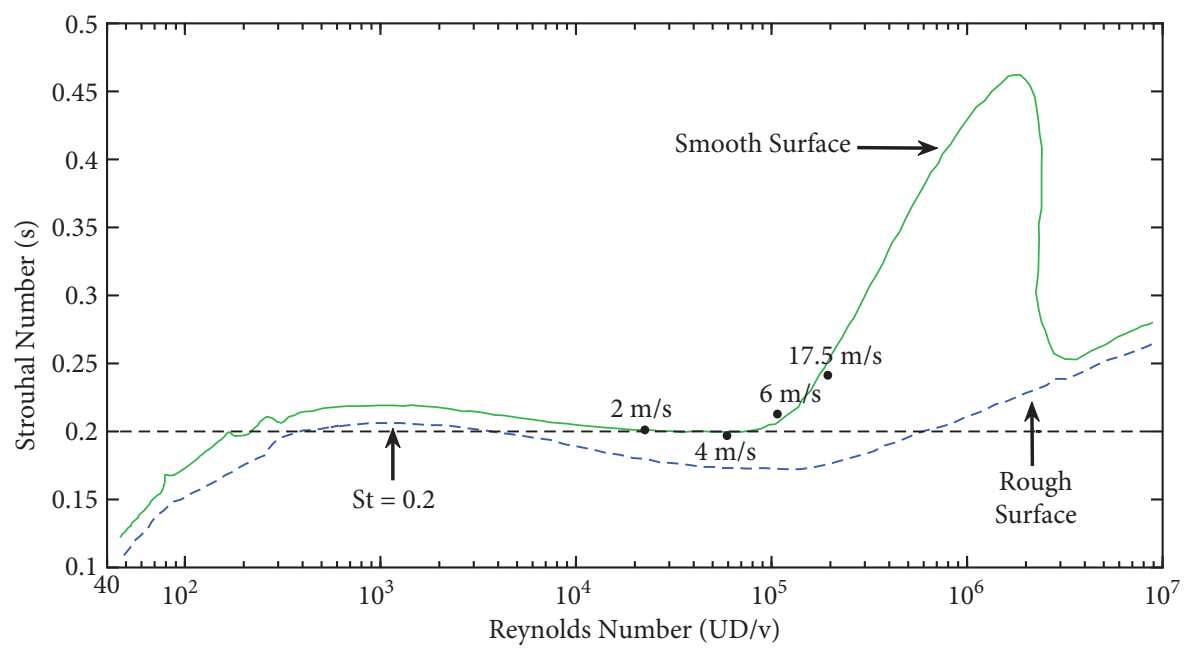

FIgURE 10: Different Reynolds numbers correspond to Strouhal numbers. 
TABLE 6: Dimensionless parameter comparison.

\begin{tabular}{lcccc}
\hline Re St & $3.2 \times 10^{4}(2 \mathrm{~m} / \mathrm{s})$ & $6.4 \times 10^{4}(4 \mathrm{~m} / \mathrm{s})$ & $9.6 \times 10^{4}(6 \mathrm{~m} / \mathrm{s})$ & $2.8 \times 10^{5}(17.5 \mathrm{~m} / \mathrm{s})$ \\
\hline St (ref 29) & 0.202 & 0.2 & 0.22 & 0.25 \\
St (simulation) & 0.2 & 0.199 & 0.227 & 0.248 \\
\hline
\end{tabular}
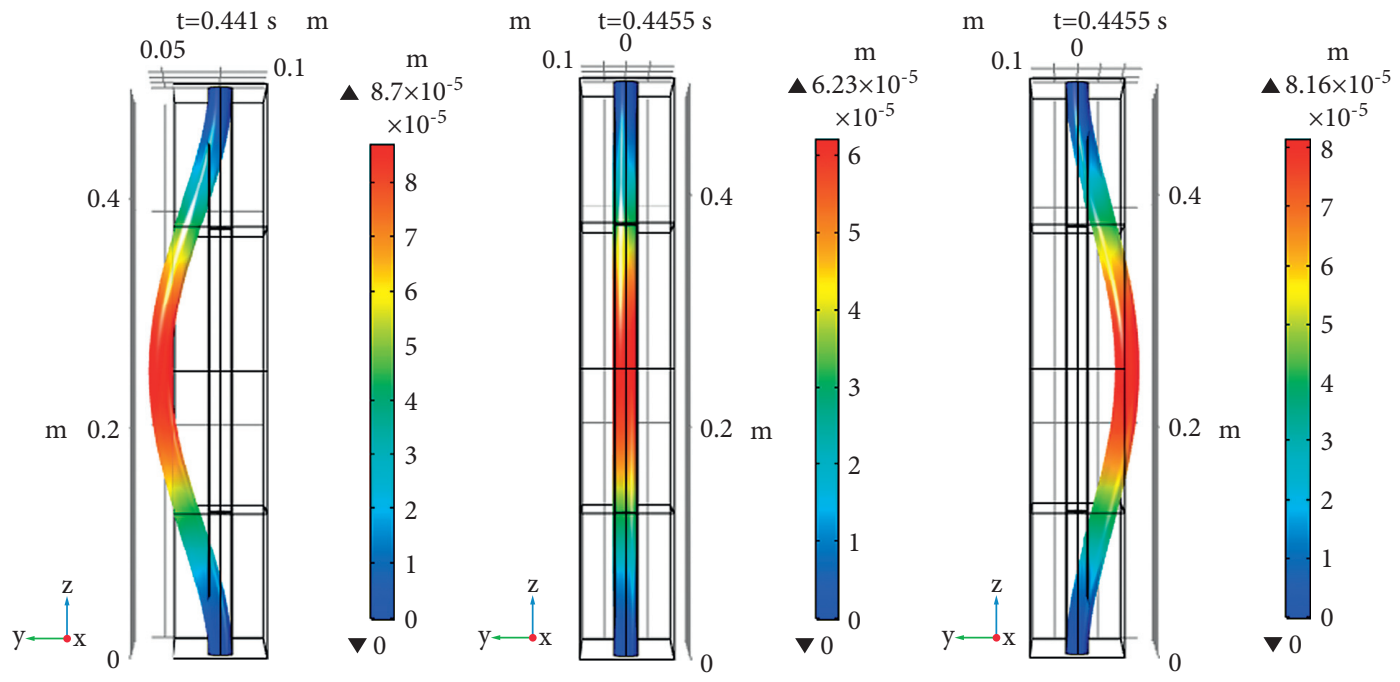

FIgURE 11: Displacement nephogram of tube bundle.

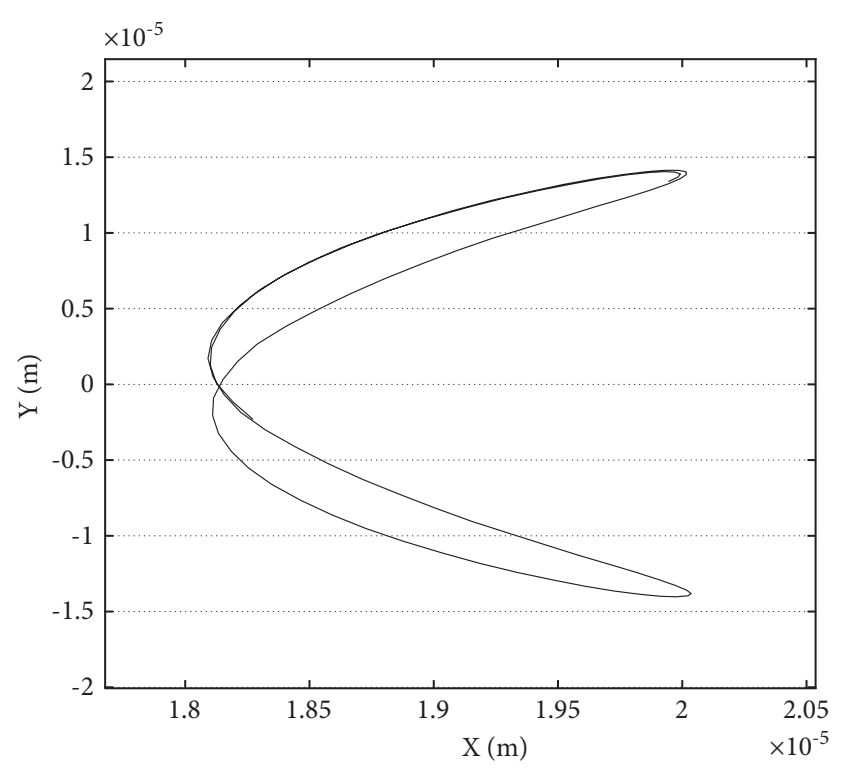

FIGURE 12: Vibration displacement curve.

According to the time-domain curves of lift and drag and the time-domain curves of displacement in $X$ and $Y$ directions in Figure 13, they are similar in their changing trends and show harmonic output after reaching a certain stable state. The frequency-domain curves of the two can be obtained by FFT transform, respectively, as shown in Figures 14 and 15.

As can be seen in Figure 14, the lift and drag frequencies are $49.75 \mathrm{~Hz}$ and $104 \mathrm{~Hz}$, respectively, which accords with the theory that the drag and lift frequency are twice. According to the $X$ and $\mathrm{Y}$ displacement frequency-domain curves, the $Y$ displacement response and $X$ displacement response correspond to the lift and drag frequencies, respectively. The $X$ displacement is mainly caused by drag, and the $Y$ displacement is mainly caused by lift.

Jiang et al. numerically analyzed the vibration response of a single row of elastic tube bundles induced by pulsating flow [30]. The research shows that vibration is mainly outof-plane vibration, and the vibration is a complex threedimensional motion. In the calculation time, the abscissa corresponds to the $X$-direction displacement, the ordinate corresponds to the $Y$-direction displacement, and the vibration displacement curve of the three-dimensional tube bundle is drawn. The obtained vibration displacement curve is shown in Figure 12. It can be found that the displacement curve presents an " 8 " shape because the frequency of $X$ direction displacement response is twice that of $Y$-direction displacement response, and the amplitude is larger. Typical Strouhal modes appear, and the displacement graph presents two symmetrical parts.

By changing the velocity in the outer basin, the vibration displacement curves at different velocities are shown in Figure 16. It can be seen that the Strouhal mode also appears at three velocities, and the amplitude of the vibration displacement curve is proportional to the velocity of external flow and increases with the increase of velocity.

3.2. Two-Way Coupling under the Action of Four-Span Tube Bundle Water. Under ideal conditions, both ends of the heat exchanger tube bundle maintain effective support, but long-term effective support does not exist, so it is necessary to analyze the flow-induced vibration of the heat exchanger tube bundle under the condition of failure support. The 

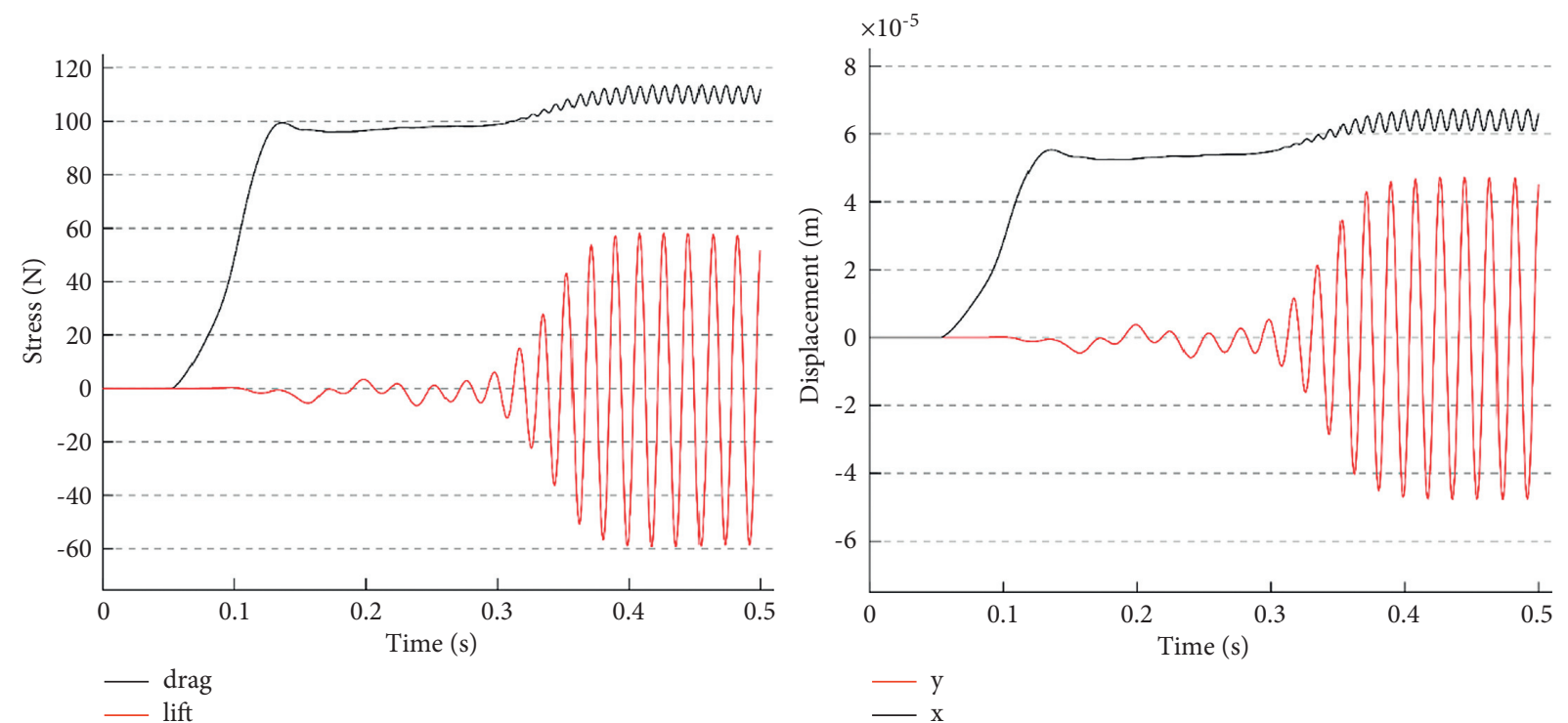

FIGURE 13: Time-domain graph of lift resistance and displacement.

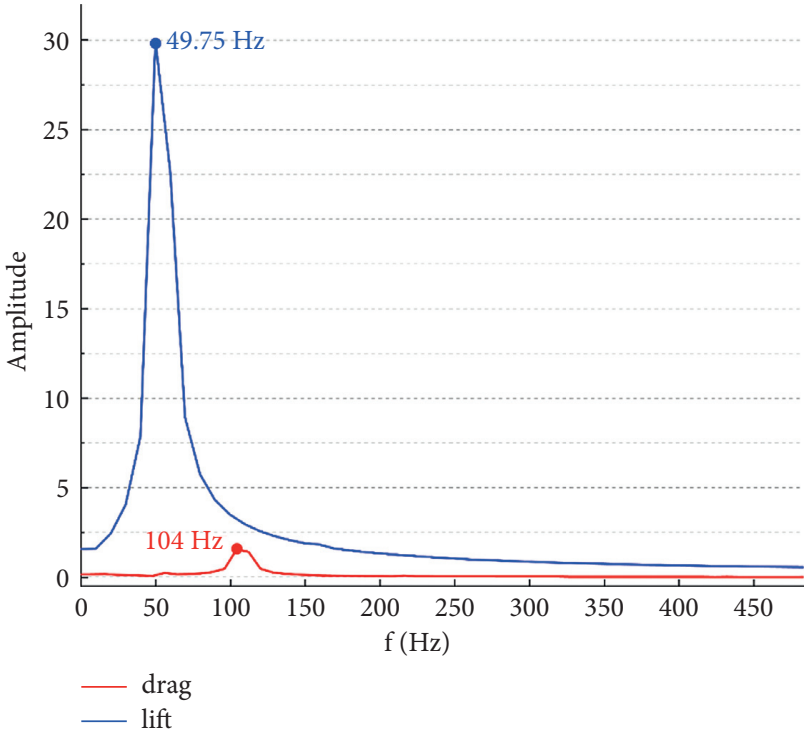

FIgURE 14: Frequency diagram of lift and drag.

single-span tube bundle is simplified as an equal four-span model, the middle baffle is regarded as a simply supported model, and the tube sheets at both ends are regarded as a fixed supported model. Each span from top to bottom is named I span, II span, III span, and IV span, respectively (see Figure 17). The four-span tube bundle is also impacted by water with a flow velocity of $4 \mathrm{~m} / \mathrm{s}$, and the vibration displacement curves of different spans are shown in Figure 18.

According to Figure 18, it is found that the vibration displacement curves of symmetrical spans are similar in " 8 " shape and the displacement amplitude is equal. The displacement amplitude of the middle two spans is larger than that of the two spans, and the middle displacement reaches

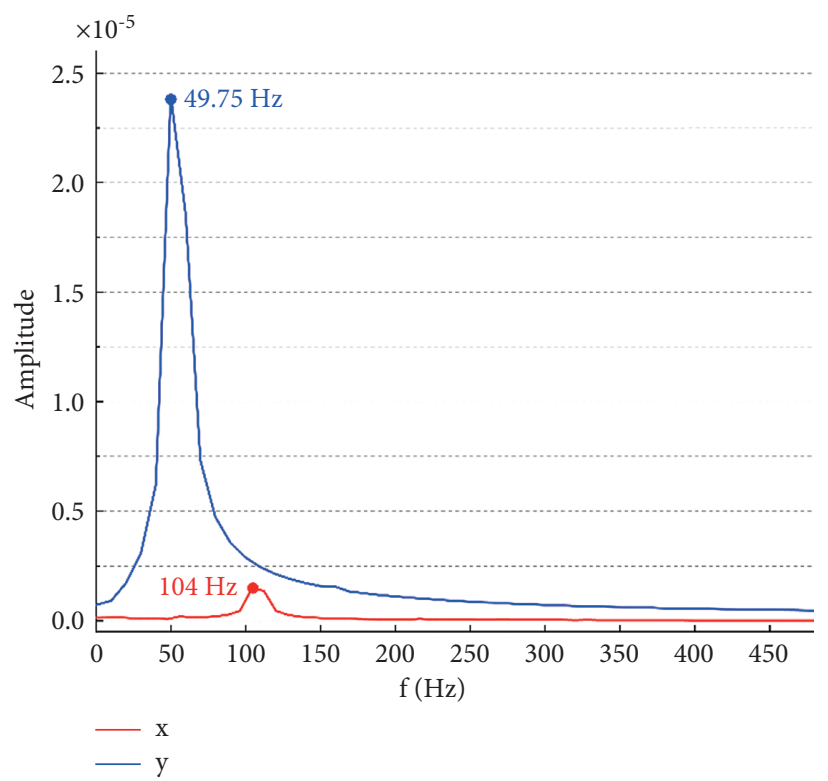

FIgURE 15: $X$ and $Y$ axial displacement response frequency curves.

the maximum. Table 7 shows the calculation results of response frequency for each span.

By comparing the data obtained in Table 7 with those in Figures 12 and 13, it can be seen that the vibration frequency for multispan tube bundle is basically the same as that of single-span tube bundle under the same velocity impact. There is a double relationship between the lift and drag frequency of the four-span tube bundle. In the process from vortex formation to shedding at the tail of the cylinder, the lift force $F_{\mathrm{y}}$ in the $Y$-direction changes from the minimum value to the maximum value and then changes to the minimum value, forming a periodic change, while the drag force $F_{\mathrm{x}}$ in the $X$-direction will form a periodic change in the process from vortex formation to shedding, and the lift force will change for two periods in the time when the drag force 


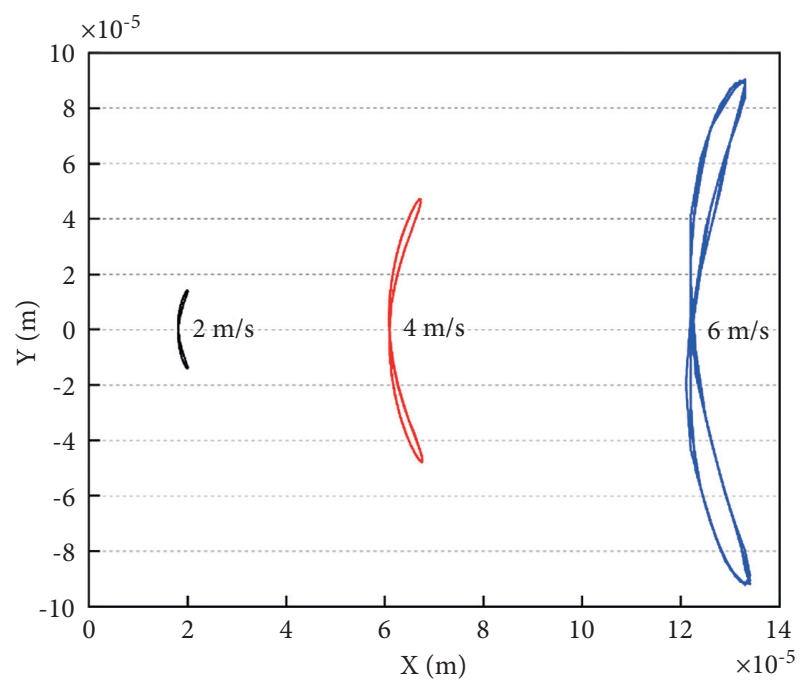

Figure 16: Vibration displacement curves at different velocities.

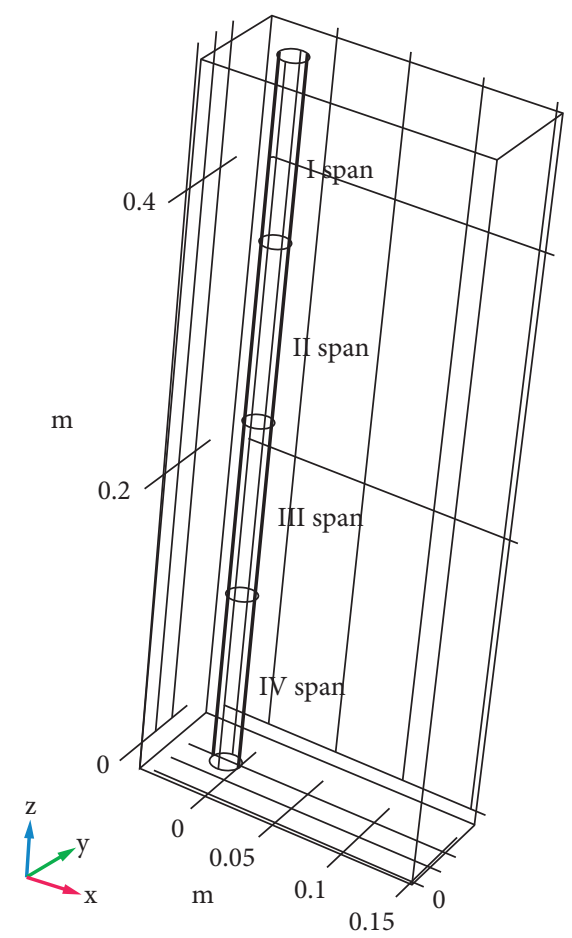

Figure 17: Four-span tube bundle model diagram.

changes for one period. There is a one-to-one corresponding relationship between displacement response frequency and lift and drag frequency.

\section{Flow-Induced Vibration of Five-Tube Bundles with Different Pitch- Diameter Ratios}

4.1. Tube Bundle with Pitch-Diameter Ratio of 1.5. Heat exchanger equipment presents large-scale production, and the tube bundle wall span presents an increasing development trend. Although the three-dimensional short tube bundle can reflect the vibration characteristics to a certain extent, it does not consider the influence of other tube bundles and has some limitations. Influenced by the distance between tubes, the flow field of each tube cannot be fully developed, and the fluids between tubes will influence and interact with each other, which leads to the difference between the flow field and stress of heat exchanger tube bundle and three-dimensional short tubes.

In order to discuss the problem of insufficient fluid development in multiple tube bundles, five tubes were selected to form a tube bundle group, and the flow field and structural displacement of the tube bundle were calculated. The influence of nodal diameter ratio on the flow field of tube bundle is discussed, and two different nodal diameter ratios of 1.5 and 3.0 with large span are modeled and meshed, respectively [31]. The model and material of each heat exchange tube are the same as those in 2.1. The total flow field length is $24 \mathrm{D}$, the center of the three-dimensional cylinder is $4 \mathrm{D}$ away from the inlet of the flow field, 20D away from the outlet of the flow field, and the width of the flow field is $8 \mathrm{D}$ [32]. Additionally, the distance between each two adjacent heat exchange tubes is $1.5 \mathrm{D}$. The calculation model is shown in Figure 19.

To validate the solution independence of the grid, the same method as in Section 3.1 is used to verify the mesh of five-tube bundles with a pitch-diameter ratio of 3.0. Four accurate grids, 217890, 241472, 303256, and 344215, were selected for evaluation. The lifting frequency and the Strouhal number of the 303256 mesh elements vary from those of the 344215 mesh elements by less than $0.8 \%$ and $1.15 \%$, respectively, as shown in Table 8 .

In order to keep a well-balanced compromise among computational time and solution accuracy, the third grid element used in the calculation domain is about 303256. Figure 20 shows the lift frequencies for different grid numbers.

There is a large velocity gradient change around the tube bundle in the whole flow field. To make the calculation results more accurate, the grid around the tube bundle is encrypted and refined. Hexahedral mesh is selected for watershed and heat exchange tube structure, the maximum element of heat exchange tube mesh is $3.29 \times 10^{-3} \mathrm{~m}$, the minimum element is $5.06 \times 10^{-5} \mathrm{~m}$, and the maximum growth rate is 1.05 . The maximum unit of basin grid is $9.36 \times 10^{-3} \mathrm{~m}$, the minimum unit is $1.01 \times 10^{-3} \mathrm{~m}$, the maximum growth rate is 1.1 , and the total number of grids is 303256. The grid division is shown in Figure 21.

The incoming velocity is $4 \mathrm{~m} / \mathrm{s}$, the turbulence $\mathrm{k}-\varepsilon$ model and transient solver are adopted, and the time step is $5 \times 10^{-4} \mathrm{~s}$. At $T=0.5055 \mathrm{~s}$, the velocity and pressure results of the XY section are shown in Figure 22.

As can be seen from Figure 22, when the outflow crosses the tube bundle transversely, the wake flows interact with each other because of the close distance between the tube bundles, and the overall flow field is more chaotic than that of a single tube.

Take a monitoring point on the middle section for the No. 5 tube, as shown in Figure 23. The vibration displacement of the tube at this point is monitored, and the 


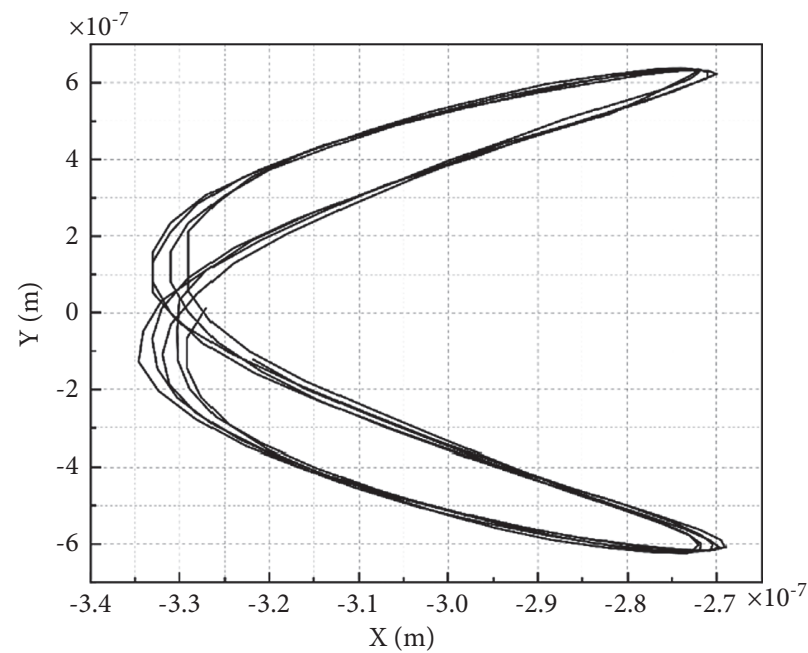

(a)

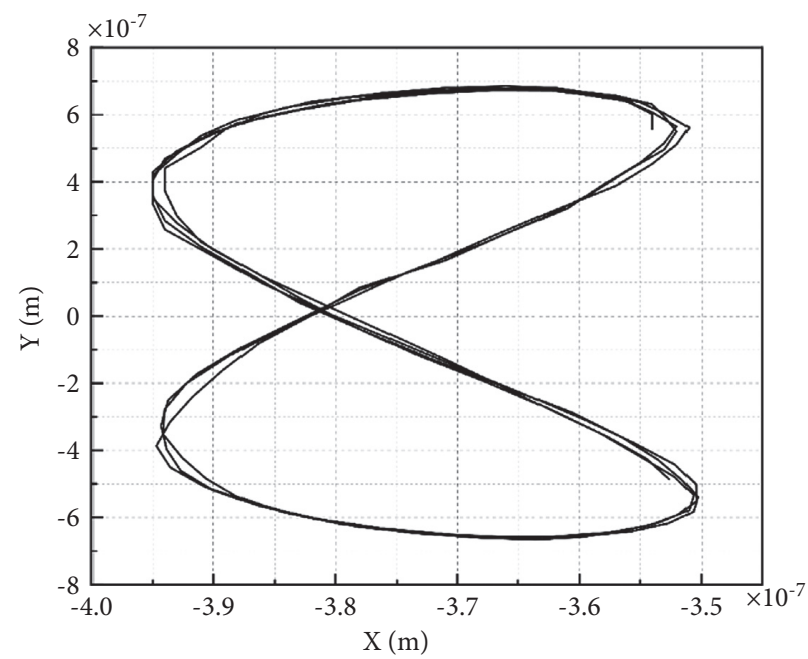

(c)

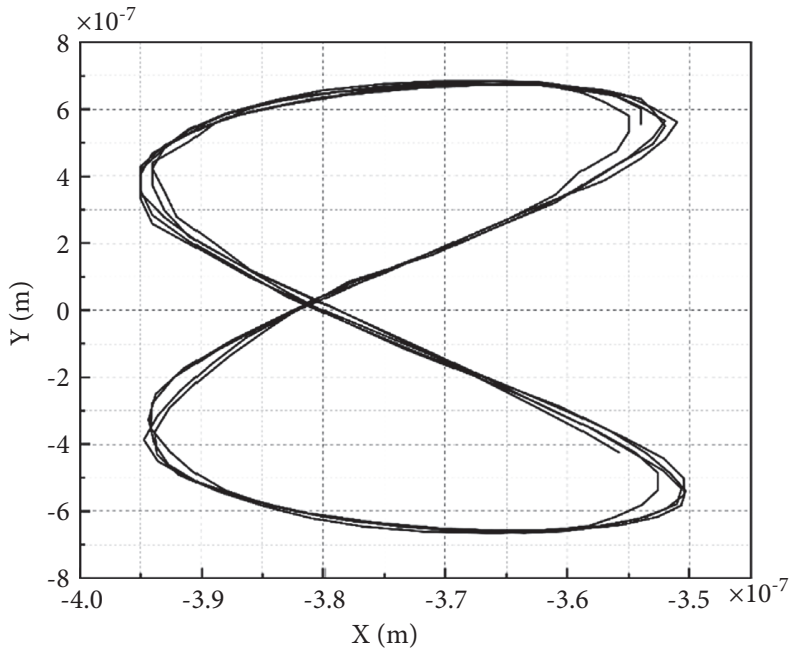

(b)

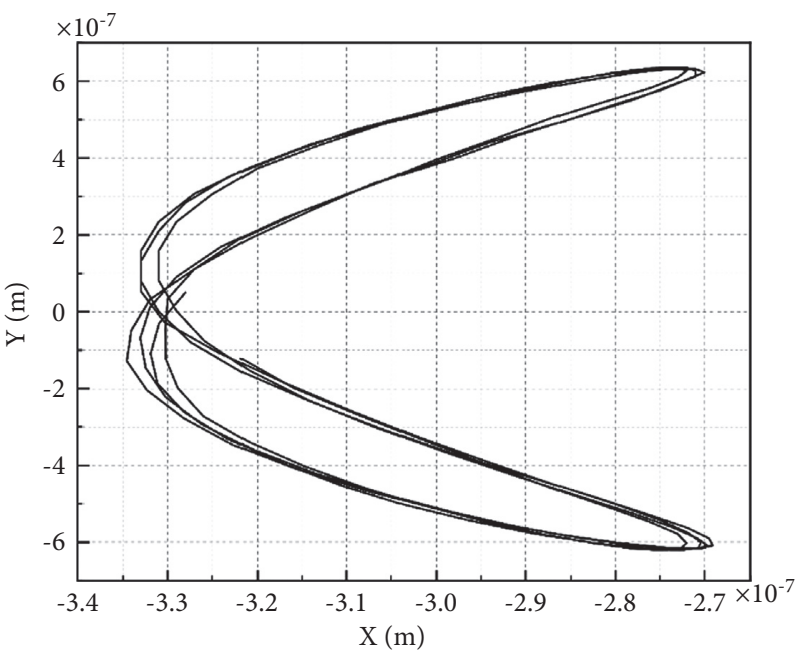

(d)

FIGURE 18: Vibration displacement curve of the four-span tube bundle. (a) I span. (b) II span. (c) III span. (d) IV span.

TABLE 7: Lift, drag frequency, and axial displacement response frequency results for each span.

\begin{tabular}{lcccc}
\hline Project & Lift frequency $(\mathrm{Hz})$ & Drag frequency $(\mathrm{Hz})$ & $Y$-direction response $(\mathrm{Hz})$ & $X$-direction response $(\mathrm{Hz})$ \\
\hline I Span & 49.17 & 99.88 & 49.82 & 99.63 \\
II span & 50.42 & 99.74 & 49.04 & 100.03 \\
III span & 49.52 & 100.01 & 49.83 & 99.02 \\
IV span & 49.99 & 99.83 & 50.03 & 99.59 \\
\hline
\end{tabular}

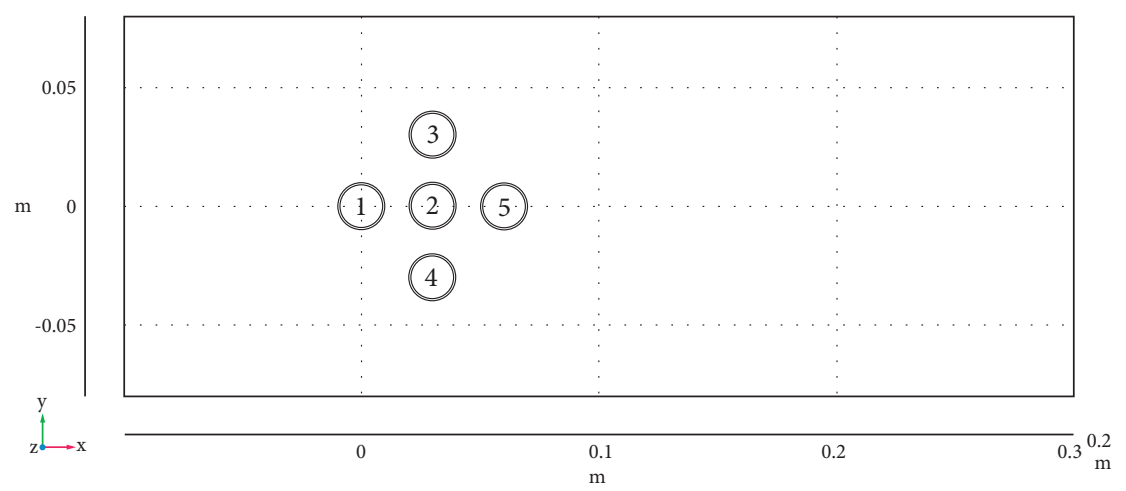

FIGURE 19: Five-tube bundle calculation model. 
TABLE 8: Grid independence results of five-tube bundle.

\begin{tabular}{lccccc}
\hline Grid & Quantity & $\overline{\mathrm{C}_{d}}$ & $\overline{\mathrm{C}_{l}}$ & Lift frequency $(\mathrm{Hz})$ & \\
\hline 1 & 217890 & 0.592 & 0.106 & 60 & 0.24 \\
2 & 241472 & 0.633 & 0.13 & 50 & 0.20 \\
3 & 303256 & 0.641 & 0.068 & 52.63 & 0.21 \\
4 & 344215 & 0.638 & 0.069 & 52.17 & 0.209 \\
\hline
\end{tabular}

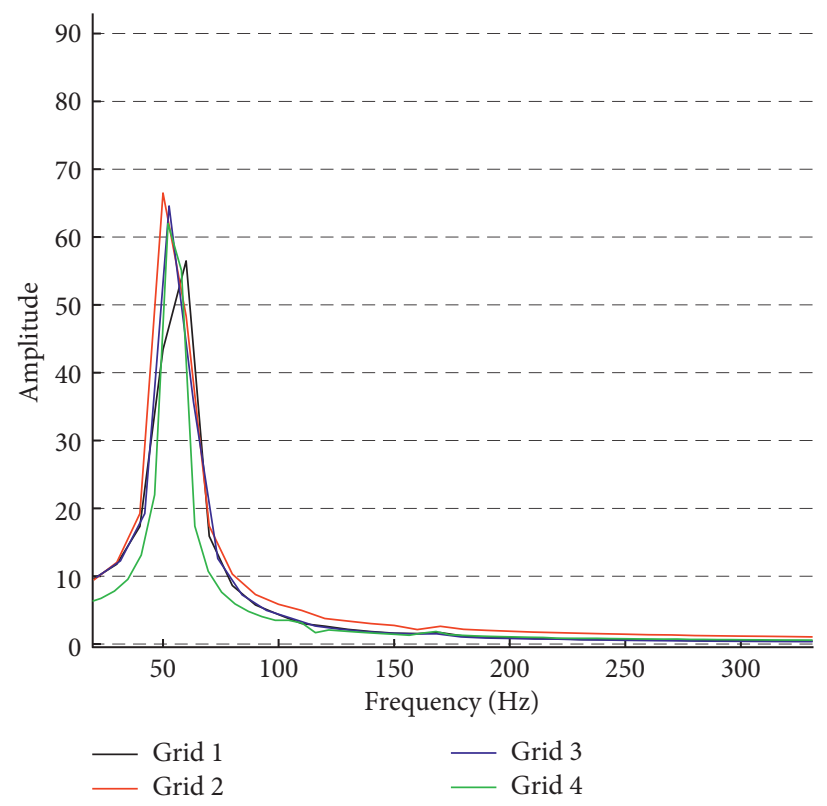

FIGURE 20: Lift frequency with different grids of five-tube bundles.

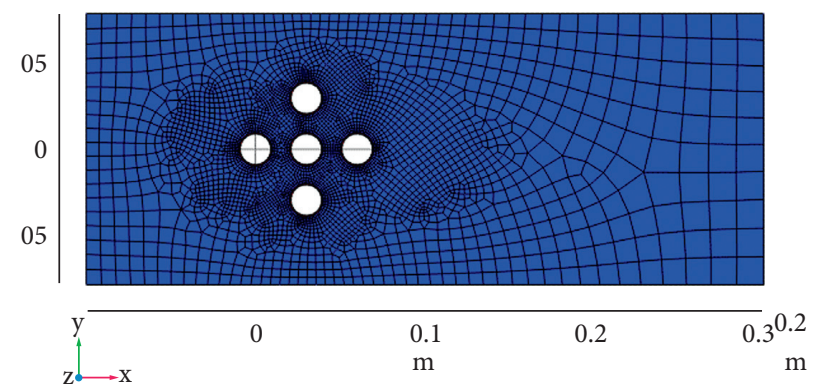

Figure 21: XY section mesh.

time-domain curve of the vibration displacement of the tube bundle shown in Figure 24 is obtained. The frequency response of the vibration displacement for the No. 5 tube can be obtained by FFT transformation of the stable part of the time-domain curve, as shown in Figure 25.

From the time-domain and frequency-domain curves, it can be seen that the wake development of the No. 5 tubes is affected by other tubes because the distance between tube bundles is too close, so the vibration frequencies in $X$ and $Y$ directions do not have a double relationship. At $73.17 \mathrm{~Hz}$, the amplitude in the $X$-direction is larger than that in the $Y$ direction. There are many peaks in the $Y$-direction lift force, while the $X$-direction drag force is gentle. The abscissa corresponds to the $X$-direction displacement, the ordinate corresponds to the $Y$-direction displacement, and the vibration displacement curve of the tube bundle is obtained, as shown in Figure 26.

Vibration displacement curves of five-tube bundles show that the Strouhal mode is not obvious because of the close distance between the tube bundles and the mutual influence of the wake of each tube bundle.

4.2. Tube Bundle with Pitch-Diameter Ratio of 3.0. When the distance between tube bundles is changed to $3 \mathrm{D}$, the watershed is unchanged, the calculation condition is the same as 4.1 , and the velocity and pressure nephogram of XY section at $T=1 \mathrm{~s}$ are obtained as shown in Figure 27.

Compared with the nodal diameter ratio of 1.5 , the wake of the tube bundle, as shown in Figure 27, has obvious vortex 


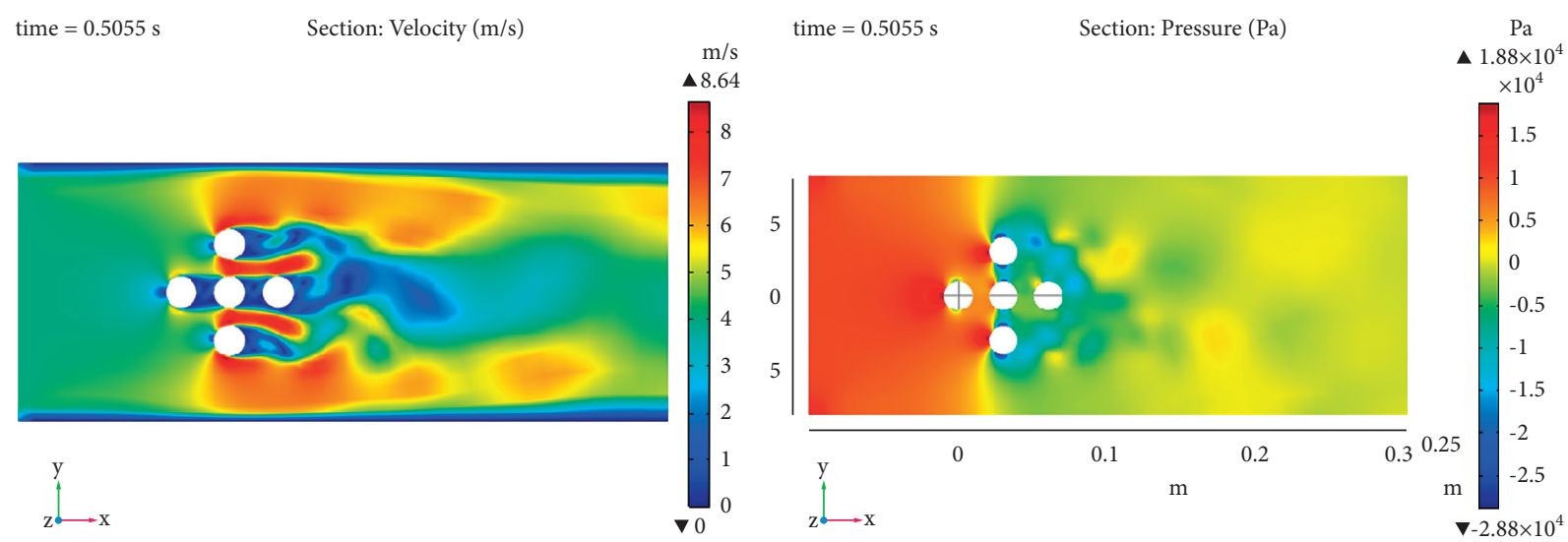

FIGURE 22: Velocity and pressure nephogram of XY section.

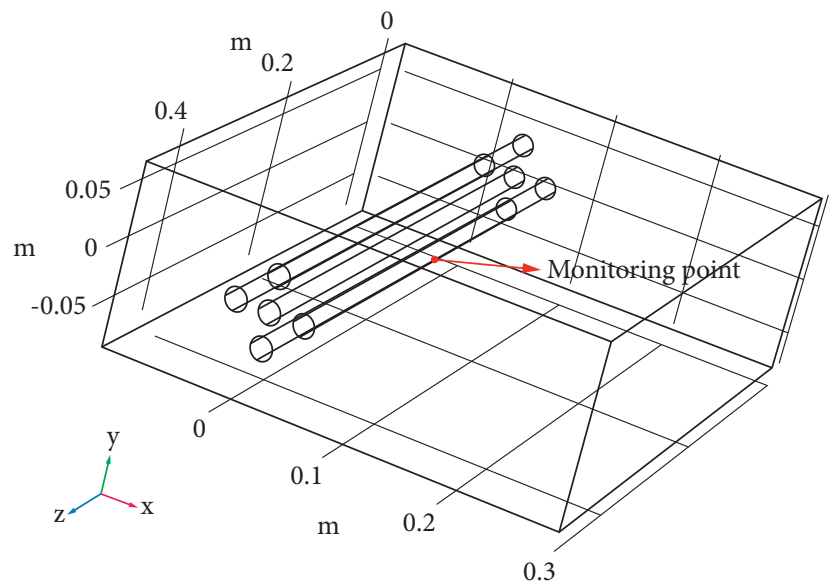

FIGURE 23: Location map of monitoring points.

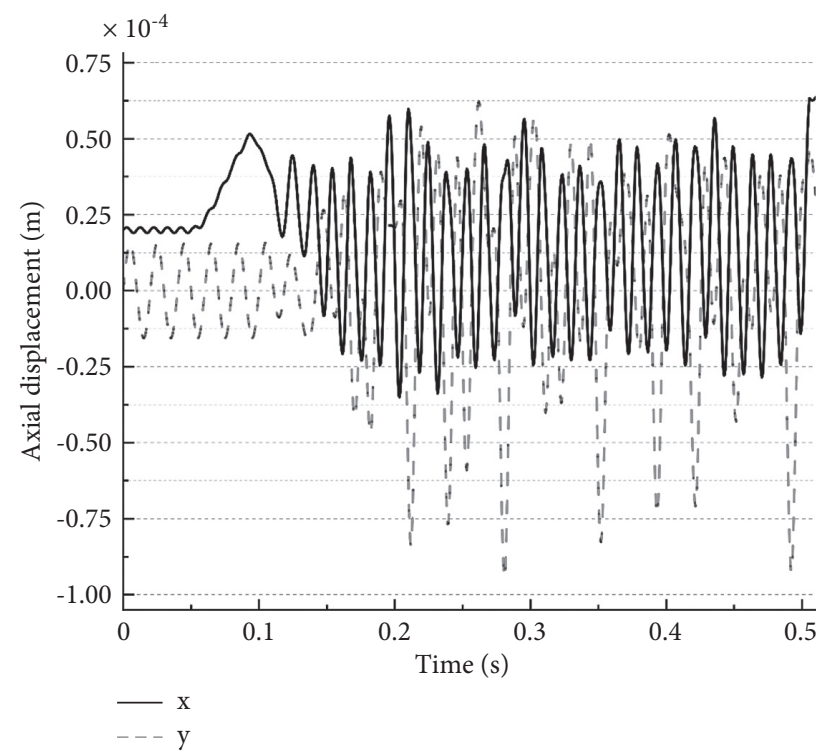

Figure 24: $X$ - and $Y$-axis vibration displacement time-domain curves.

shedding. Take the same monitoring point on the No. 5 tube, and get the vibration displacement curve of the tube bundle at this point, as shown in Figure 28. The frequency-domain curve of the tube is obtained by FFT transformation of the relatively stable part in the time domain, as shown in Figure 29. 


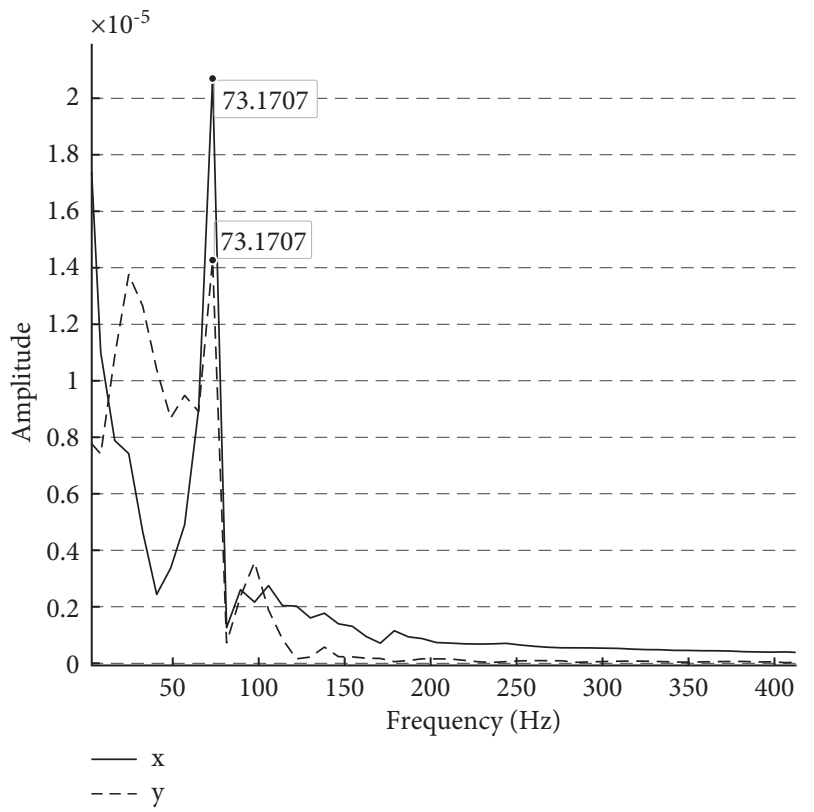

FIgURE 25: $X$ - and $Y$-axis vibration displacement frequency-domain curves.

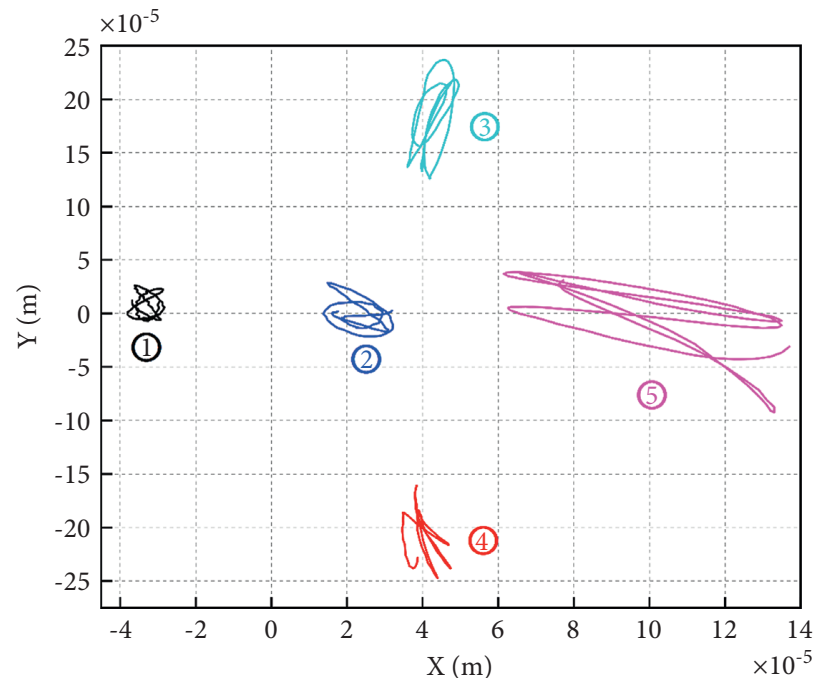

FiguRE 26: Vibration displacement curves of different tube bundles with the pitch-diameter ratio of 1.5.

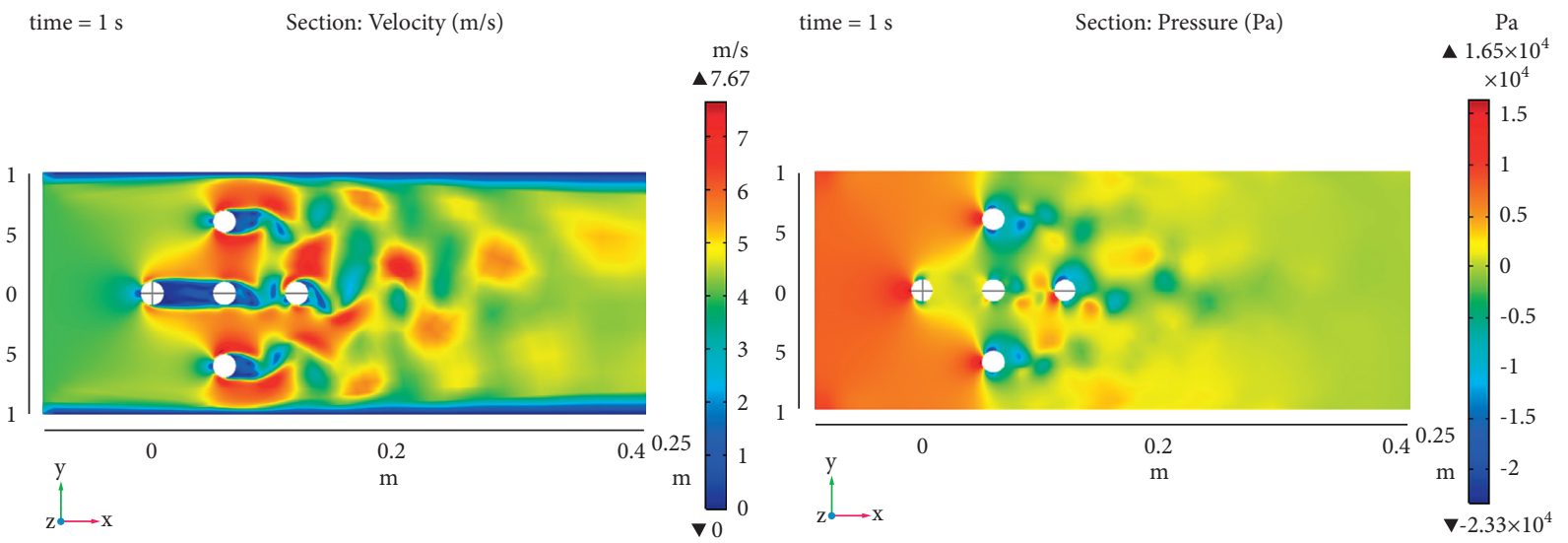

FIgURE 27: Velocity and pressure nephogram of XY section. 


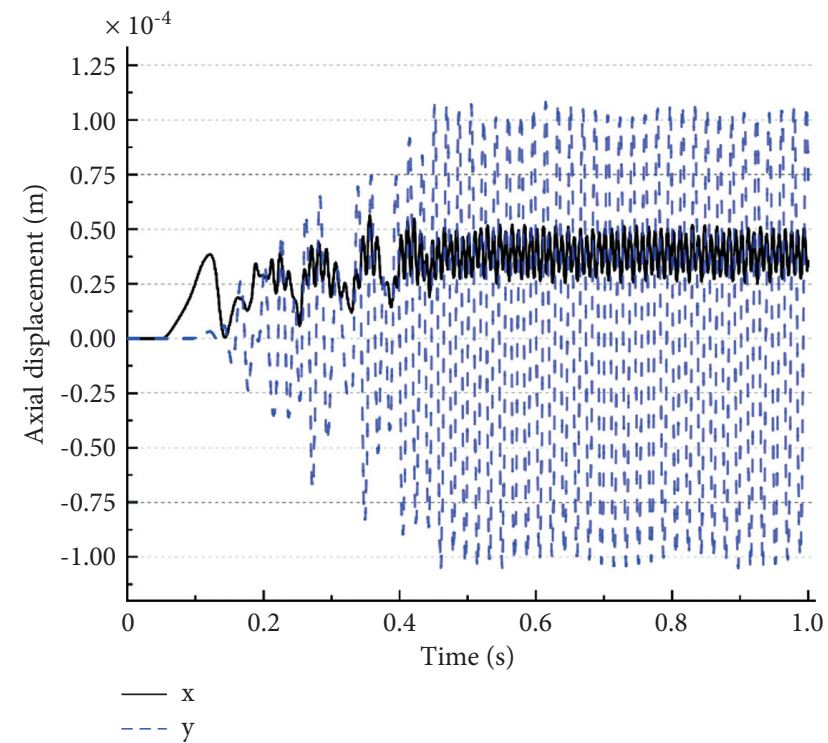

Figure 28: $X$ - and $Y$-axis vibration displacement time-domain curves.

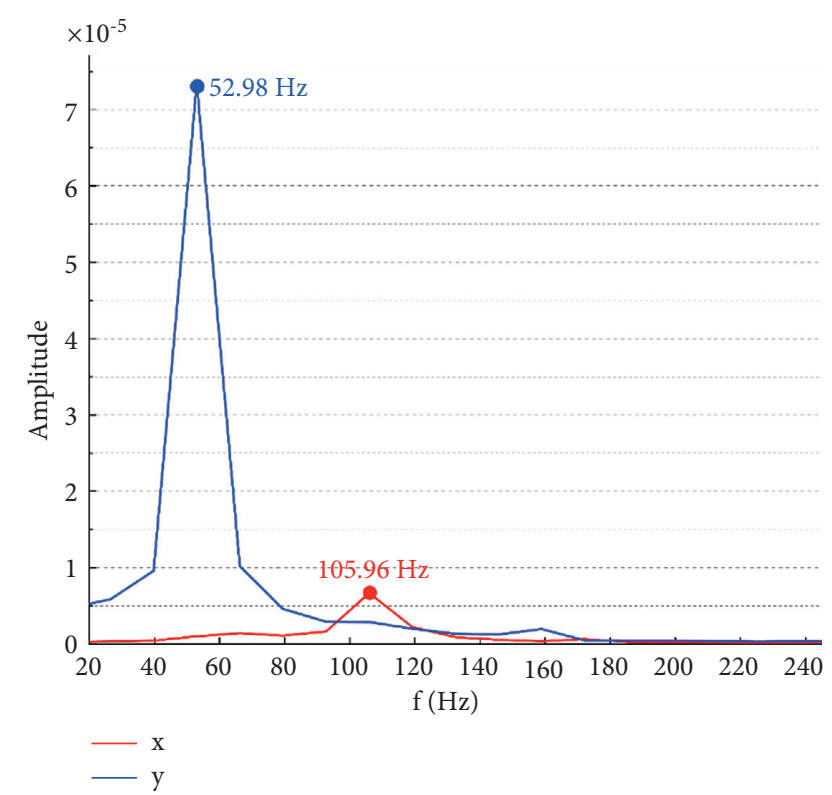

Figure 29: $X$ - and $Y$-axis vibration displacement frequency-domain curves.

From the time-frequency-domain curves, it can be seen that the vibration is more stable than that when the pitchdiameter ratio is 1.5 due to the larger distance between tube bundles, and the frequency response of $X$-direction and $Y$ direction vibration displacement presents a double relationship. The abscissa corresponds to the $X$-direction displacement, and the ordinate corresponds to the $Y$-direction displacement to obtain the vibration displacement curve, as shown in Figure 30.

It can be found that as the distance between the tubes increases, the influence of the wake between the tubes decreases. Compared with the vibration displacement curve when the nodal diameter ratio is 1.5 , the vibration

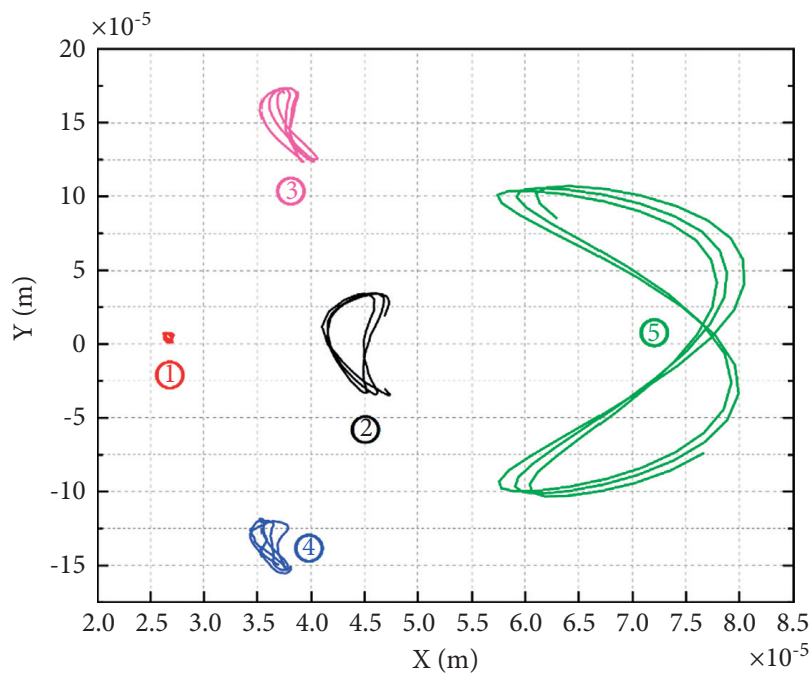

Figure 30: Vibration displacement curves of different tube bundles with the pitch-diameter ratio of 3.0.

displacement curve presents obvious Strouhal mode. No. 5 tube bundle forms a complete vortex and falls off, and the wake is hardly affected. Its vibration displacement curve is a regular " 8 " shape, showing a typical Strouhal mode shape.

\section{Conclusions}

In this paper, the heat exchanger tube bundle is taken as the research object. By comparing the theoretical calculation value of the natural frequency of the heat exchanger tube with the numerical simulation calculation, the calculation error is about $3 \%$, which verifies that the calculation result is effective. The flow-induced vibration characteristics of single-span and multispan under the condition of failure support and the flowinduced vibration characteristics of five-tube bundles with two different pitch-diameter ratios (1.5 and 3.0) are simulated and analyzed. The results show the following:

(1) The natural frequency of multispan tubes decreases with the increase of the number of support failures.

(2) The vibration frequency of multispan tubes is basically the same as that of single-span tubes, and the failure support does not change the vibration frequency of tube bundle.

(3) The displacement curves of single-span and multispan tubes show an " 8 " shape and typical Strouhal mode, and the amplitude increases with the increase of fluid velocity. The " 8 "-shaped curves having a left half that is a perfect mirror image of the right half spans multispan tube bundle is similar, and the displacement amplitudes are equal.

(4) When the pitch-diameter ratio is 3.0, the influence of the fluid between the tubes becomes smaller due to the increase of the tube bundle spacing, which makes the vibration more stable. At this time, the frequency response of vibration displacement presents a double relationship, and the vibration displacement curve presents an obvious Strouhal mode. 


\section{Data Availability}

The data used to support the findings of this study are available from the corresponding author upon request.

\section{Conflicts of Interest}

The authors declare that they have no conflicts of interest.

\section{Acknowledgments}

This paper was supported by the Stable Supporting Fund of Acoustic Science and Technology Laboratory (JCKYS2020604SSJS013), the National Natural Science Foundation of China (11704090), University Nursing Program for Young Scholars with Creative Talents in Heilongiiang Province (UNPYSCT-2018207), and the project of Nature Scientific Foundation of Heilongjiang Province (LH2020A016).

\section{References}

[1] S. Kosai and H. Unesaki, "Quantitative analysis on the impact of nuclear energy supply disruption on electricity supply security," Applied Energy, vol. 208, pp. 1198-1207, 2018.

[2] Y. Chen and X. D. Chen, "Technology development of largescale heat exchanger in China," Journal of Mechanical Engineering, vol. 49, no. 10, pp. 134-163, 2013.

[3] Q. D. Nie, "Technical progress of pressure vessel," Pressure Vessel, vol. 6, pp. 81-84, 1986.

[4] Z. H. Chen, "Vibration calculation of PTA feed preheater and stability analysis of tube bundle," China Boiler and Pressure Vessel Safety, vol. 20, no. 1, pp. 41-43, 2004.

[5] Z. H. Jin, W. Jin, W. J. Wang, B. Gong, and L. Cong, "Application of vibration analysis in shell-and-tube heat exchanger design," Journal of Shenyang Institute of Chemical Technology, vol. 16, no. 1, pp. 57-60, 2001.

[6] A. S. El-Amoush and S. Affijad, "Hydrogen induced cracking and pitting of brass heat exchanger tube," Materials Science and Technology, vol. 24, no. 6, pp. 711-717, 2008.

[7] J. X. Wu, Q. W. Dong, S. M. Liu, and Z. W. Wang, "Failure analysis, prevention, and online detection of shell-and-tube heat exchanger," Pressure Vessel, vol. 6, pp. 57-60+0, 2001.

[8] J. Z. Wang, Fluid-induced Vibration Failure Analysis of Heat Exchanger Based on Weak Fluid-Structure Coupling Method, East China University of Science and Technology, Shanghai, China, 2011.

[9] M. Yang, J. J. Ye, K. Zou, and G. Z. Dong, "Numerical simulation of unsteady periodic fully developed flow across a tube bundle," Journal of Xi'an Jiaotong University, vol. 11, pp. 1122-1125, 2001.

[10] S. Y. Chen, "Vibration analysis of heat exchanger tube bundle," Lanhua Science and Technology, vol. 14, no. 4, pp. 63-66, 1996.

[11] T. Zeng, "Heat transfer enhancement of shell-and-tube heat exchanger and vibration of tube bundle," Chemical Equipment Technology, vol. 3, pp. 25-26, 2000.

[12] P. L. Dong, Force and Stress Analysis of Tube Bundle of Elastic Tube Bundle Heat Exchanger, Shandong University, Shandong, China, 2016.

[13] Q. D. Nie and W. Tan, Fluid-induced Vibration of Shell-AndTube Heat Exchanger, Sinopec Press, Beijing, China, 2014.

[14] NRC, SAN ONOFRE Nuclear Generating Station-NRC Augmented Inspection Team Report, United States Nuclear Regulatory Commission, Rockville, MA, US, 2012.
[15] Y. X. Lai, Dynamic Characteristics Analysis and Fluid-Induced Vibration Study of Heat Exchanger Tube Bundle, Nanjing University of Technology, Nanjing, China, 2006.

[16] W. Tan, W. J. Jin, H. Wu, and Z. Yang, "Study on the influence of rigid pipes on the hydroelastic instability of square tube bundles," Pressure Vessels, vol. 33, no. 3, pp. 1-8, 2016.

[17] Y. Zhu, Numerical Simulation of Two-Way Fluid-Structure Coupling Mechanism of Fluid-Induced Vibration of Heat Exchange Tubes Filled with Internal and External Liquid, Nanchang University, Nanchang, Chian, 2015.

[18] W. Zhao, F. Xue, G. Shu et al., "Analysis of flow-induced vibration of steam generator tubes subjected to cross flow," Nuclear Engineering and Design, vol. 275, pp. 375-381, 2014.

[19] J. W. Liu, H. Y. Guo, and J. Zhao, "Numerical simulation of flow around tandem double cylinders with equal diameter," Journal of Ocean University of China, vol. 43, no. 12, pp. 92-97, 2013.

[20] Z. P. Feng, F. G. Zang, and Y. X. Zhang, "Study on flowinduced vibration characteristics of tube bundle structure," Atomic Energy Science and Technology, vol. 49, no. 1, pp. 51-57, 2015.

[21] C. Y. Zhou, R. M. C. So, and K. Lam, "Vortex-induced vibrations of an elastic circular cylinder," Journal of Fluids and Structures, vol. 13, no. 2, pp. 165-189, 1999.

[22] J. Liu, C. Huang, and N. B. Jiang, "Unsteady model for transverse fluid elastic instability of heat exchange tube bundle," Mathematical Problems in Engineering, vol. 2014, Article ID 942508, 7 pages, 2014.

[23] Y. F. Zhang, B. N. Shou, J. Zou, J. Zhu, S. Li, and Y. Zhang, Heat Exchangers, China Standards Press, Beijing, China, 2014.

[24] J. Zhang, Z. Liang, and C. J. Han, "Fluid-structure coupling analysis of U-shaped liquid-filled pipeline," Journal of Applied Mechanics, vol. 32, no. 1, pp. 64-68, 2015.

[25] W. Tan, Z. Li, Y. P. Wang, and Z. B. Jia, "Study on the influence of support failure on vibration characteristics of baffled rod heat exchanger tube bundle," Chemical Machinery, vol. 45, no. 3, pp. 282-286, 2018.

[26] Q. Zhang, Study on Stochastic Characteristics of Hydroelastic Instability of Heat Exchanger Tube Bundle, Harbin Engineering University, Harbin, China, 2014.

[27] J. T. Xing, S. Zhou, and E. J. Cui, "Overview of fluidstructure coupling mechanics," Advances in Mechanics, vol. 1, pp. 20-39, 1997.

[28] K. Shahab and A. N. Luqman, "Experimental study on crossflow induced vibrations in heat exchanger tube bundle," China Ocean Engineering, vol. 31, no. 1, pp. 91-97, 2017.

[29] S. S. Chen, Flow-induced Vibration of Circular Cylindrical Structures, Hemisphere Publishing Corp, Washington, DC, USA, 1987.

[30] B. Jiang, M. C. Tian, X. L. Leng, Y. Tang, and L. Cheng, "Fluidstructure coupling analysis of fluid-induced vibration of elastic tube bundles," in Proceedings of the 9th National Conference on Hydrodynamics and the 22nd National Symposium on Hydrodynamics, Ocean Mountain Edition, Beijing, China, 2009.

[31] Y. Q. Huang, J. Deng, and A. L. Ren, "Research on lift and drag in unsteady viscous flow around circular cylinders," Journal of Zhejiang University (Science Edition), vol. 1, pp. 100-105, 2003.

[32] Y. Y. Fang, "Flow field size selection and mesh generation of 3D numerical simulation of flow around a circular cylinder," Journal of Waterway and Harbor, vol. 30, no. 1, pp. 70-76, 2009. 